\title{
Calcul économique des réseaux de distribution d'eau
}

\section{Economic computation of water supply nets}

\author{
Par Shrigua Irimar (*),
}

A. I. Lg.

Professeur associé. directeur de la Division Hydrotechnique, a l'Israel Institute of Technology, Haifa, Israël, président de la Section d'Hydrologie, Inion Géodésique el Géophysique d'Istaëi.

In réseau de distribution d'eau pose un problème indéterminé hydrauliquement, mais soInble univoquement en introduisant la condition d'économie, minimum des capitıx investis on des dépenses annuelles.

L'analyse des divers éĺments permet de les évaluer en fonction des diametres des conduites et de la hauleur du réservoir sous forme de paraboles d'ordre n. Le problème exige la solution d'un minimum relatif à plusieurs inconnues, parmi lesquelles les distanees de l'origint aux points oi le diametre change brusquement. Le diametre économique de la conduite de refoulement varie à peu près comme la racine carrée du débit. ce qui définit une milesse íconomique presque indépendante du debit.

Le diamètre initial de le conduile principale est déterminé à partir de la perte de charge disponible et du débit initial supposé constant le long de la conduite. Les diamitres suivants résullent d'une construction graphique très simple, dite «méthode de la parabole ». La hautenr du réservoir en déconle et des corrections sont envisagées. La solution économique exige une ligne d'énergie concave vers le haut, et des vitesses variant à peu près comme les pentes piézométriques on comme $D^{1,5}$. La hauteur économique varip $\dot{a}$ pen près linéairement comme la longueur de la conduite.

Diverses dispositions topographiques sont étudiées, ainsi que la sifuation du réservoir.

Le cas de résernar ramifiés et maillès est envisagé.
Planning of a uater supply distribution net is " hydrantically indelerminate problem, yet uniquely solved bly introducing the condition of economy, minimum of capital investment or of annual expenditure.

The analysis of the barious elements leads to parabolic functions of degree $\mathrm{n}$ of pipe diameters and reserwoir height. The problem requires the solution of a relatioe minimum in many unknowns, among which the abscissat of the points of sudden change in pipe dimmeter. The economical diameter of the delinery pipe naries approximately as the square rool of the discharge, which defines an economical polocity almost independent of the rate of flow.

The initial diameter of ller main is delermined by the abailable Ioss of head and the initial discharge assumed constant along the main. The following diameters resull from a nery simple graphical 'parabolic' method. The reservoir height is deduced therefrom and corrections considered. The economical solution requires an energy line concale upuards, belocities barying approximately as the hydraulit: gradient or as D1.t. The economiral height varies approximately lincarly wifh the lengfh of the main.

Various topographical outlays are considered, as well as a resermoir site on a hillside.

The effect of brathing and complex nets is studied.
Afin de rendre service à nos lecteurs, une version anglaise, raccourcie, accompagne en bas de page le texte français. Les formules ne sont pas répétées, mais elles sont remplacées par un numéro (1) correspondant à celui qu'elles portent dans le texte français.
To assist our readers, a condensed English version is given at the foot of the page. Formulae are not repeated but the numbers (1) which replace them correspond to those accompanying each formula in the French text. 


\section{LISTE DES SYMBOLES}

$A_{k}$ : nœud où le diamètre change de $\mathrm{D}_{k-1}$ en $\mathrm{D}_{k}$.

$A_{0}$ : début de la conduite principale.

$A_{n}$ : terminaison de la conduite principale. $a, a_{1}, a_{2}$ : coefficients de prix (conduites, poste de pompage, réservoir).

$B_{k}:$ branchement d'une conduite secondaire; coefficient numérique.

$b, b_{1}, b_{2}$ : coefficients de prix (conduites, poste de pompage, réservoir).

C : coefficient de la formule de HAzENWiLLIANS.

$\mathrm{C}_{k}$ : coefficient numérique (débit).

D), $D_{1}$ : diamitre de la conduite principale.

1) : diametre de la conduite principale, troncon initial.

$\mathrm{D}_{k}$ : diamctre de la conduite principale, tronçon $\mathbf{A}_{k-1} \mathbf{A}_{k}$.

$\left.\overline{\mathrm{D}}_{l: \cdots} \ldots(\mathrm{I})_{k-1}+\mathrm{D}_{k i}\right) / 2$.

$\mathrm{D}^{\prime}, \mathrm{D}^{\prime \prime}$ : diametre d'une conduite secondaire.

$\mathrm{D}^{\prime}{ }_{0}, \mathrm{D}^{\prime \prime}{ }_{0}$ : diametre d'une conduite secondaire, tronçon initial (minimum des dépenses annuelles).

$\mathrm{D}_{r}$ : diamètre de la conduite de refoulement (minimum des capitaux).

$\mathbf{D}_{r}^{\prime}$ : diamètre de la conduite de refoulement (minimum des dépenses annuelles).

$e$ : prix de l'énergie (par ligm).

$\mathrm{F}$ : fonction auxiliaire.

$f$ : coefficient de frottement (des tuyaux).

$G$ : fonction auxiliaire.

$\mathrm{G}_{k}$ : coefficient numérique (débit).

$\mathrm{H}, \mathrm{H}_{0}$ : hauteur du fond du réservoir au-dessus du sol (minimum de W).

$\mathrm{H}^{\prime}$ : hauteur du fond du réservoir au-dessus du sol (minimum de $\mathrm{U}$ ).

$h_{o}$ : hauleur de la conduite de refoulement an-dessus du fond de réservoir.

$h_{r}$ : hauleur de pompage.

$h_{s}$ : hauteur du pied du réservoir au-dessus de la nappe aquifère.

J : pente de la ligne d'énergie, conduite principale.

$J_{0}$ : pente de la ligne d'énergie, conduite principale (début).

$J_{k}$ : pente de la ligne d'énergie, conduite principale (troneon $\mathrm{D}_{k}$ ).
$\mathrm{J}_{0}$ : pente de la ligne d'énergie, conduite principale (début, minimum de U).

$\overline{\mathbf{J}}_{k}$ : pente de la ligne d'énergie, conduite principale (correspond à $\mathrm{Q}_{k}$ ).

$J^{\prime}, J^{\prime \prime}$ : pente de la ligne d'énergie, conduite secondaire.

$\mathrm{J}_{r}$ : pente de la ligne d'énergie, conduite de refoulement.

$\mathbf{J}_{r r}, \mathbf{J}_{r r}^{\prime}$ : pente critique économique (minimum de $W, U)$.

$j$ : pente movenne du sol.

$\mathrm{K}$ : coefficient de la porte d'énergie.

$\mathrm{L}, \mathrm{I}_{\mathrm{A}_{\mathrm{I}}}$ : Iongueur totale de la conduite principale.

$L^{\prime}, L^{\prime \prime}$ : longueur totale d'une conduite secondaire.

$\mathbf{L}_{r}$ : longueur totale de la conduite de refoulement.

$\mathbf{L}_{t}$ : longueur totale d'une boucle.

$l, l_{k}$ : longueur d'un troncon de conduite principale.

$l^{\prime}$ : longueur d'un troncon de conduite secondaire.

$m=1,852$ : indice de puissance de Q (perte d'énergie).

$\mathbf{N}$ : puissance nominale du groupe motopompe.

$n$ : nombre des troncons de la conduite principale;

coefficient de puissance (de Q).

$n^{\prime}$ : nombre des troncons d'une conduite secondaire.

$\mathrm{P}, \mathrm{P}_{\mathrm{I}}$ : prix par mètre courant de la conduite principale.

$\mathrm{P}^{\prime}, \mathrm{P}^{\prime \prime}$ : prix par mètre courant d'une conduite secondaire.

$P_{0}$ : prix par mètre courant de la conduite principale, tronçon initial.

$\mathbf{P}_{k}$ : prix par mètre courant de la conduite principale, troncon $\mathrm{D}_{k}$.

$P_{r}$ : prix par mètre courant de la conduite de refoulement.

$p_{j}$ : taux annuel (entretien, reparation, assurance).

$Q, Q_{1}$ : débit dans la conduite principale.

$Q_{n}$ : débit dans la conduite principale (début).

$\mathrm{Q}_{k}$ : débit dans la conduite principalc (en $\mathbf{A}_{k}$ ). 
$Q_{i-}$ : débit dans la conduile principale (avant le noud $B_{i}$ ).

$Q_{i+}$ : débit dans la conduite principale (après le nœud $B_{i}$ ).

$\overline{Q_{k}}=\left(\mathrm{Q}_{k}+\mathrm{Q}_{k+1}\right) / 2$.

$Q_{r}$ : débit dans la conduite de refoulement. $Q^{\prime}, Q^{\prime \prime}$ : débit dans une conduite secondaire. $Q^{\prime}{ }_{1}, Q_{0}^{\prime \prime}$ : débit dans une conduite secondaire (début).

$q_{i}$ : débit du branchement (en $B_{i}$ ).

R : coefficient numérique (des débits).

$r$ : taux d'intérêt de $W$.

$r_{j}:$ taux d'amortissement de $\mathrm{W}_{j}$.

$\mathrm{T}_{j}$ : durée de vie d'un élément $j$.

$l$ : temps de fonctionnement annuel de la pompe.

$\mathrm{U}$ : dépenses annuelles, lotales.

$\mathrm{U}_{1}$ : dépenses amnuelles, intérêt.

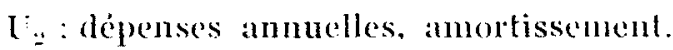

$\mathrm{l}_{; 3}$ : dépenses annuelles, contretien, réparations, assurance.

$\mathrm{U}_{4}$ : depenses annuelles, pompage.

$\mathrm{U}_{5}$ : dépenses annuelles, fixes,

$\mathrm{U}_{6}$ : dépenses annuelles, annuité (remboursement des capitaux).

$u=0,447$ puissance numérique.

$v$ : vitesse moyenne dans la conduite principale.

$v_{r}$ : vitesse économique dans la conduite de refoulement.

W : capital, total.

$\mathrm{W}_{1}$ : capital, poste de pompage.

$\mathrm{W}_{\mathrm{a}}$ : capital, réservoir d'eau.

$\mathrm{W}_{3}$ : capital, conduile de refoulement.

$\mathrm{W}_{4}$ : capital, conduite principale.

$W_{i n}$ : capital, conduiles secondaires.

$W_{6}$ : capital, captage el installations auxiliaires.

$W_{i}$ : capital, dépenses initiales, etc...

$\mathrm{X}$ : abscisse numérique.

$x_{k}$ : abscisse du noud $A_{k}$ où $D_{k \cdots 1}$ change en $\mathrm{D}_{k}(k=0,1 \ldots, n-1)$.
$x_{n}=\mathrm{D}_{r}$ : diametre de la conduile de refoulement.

$x_{n+1}=\mathrm{H}$ : hauleur du fond de réservoir au-dessus du sol.

$x_{n: 2}=\mathrm{D}_{0}$ : diametre initial de la conduite principale.

$y, y_{1}:$ perte d'énergie dans la conduite principale.

$y^{\prime}, y^{\prime \prime}$ : perte d'énergie dans une conduite s'condaire.

$y_{r}$ : perte d'éncruite dans la conduite de refoulement.

$z$ : denivellation du sol le long de la conduite principale, ou ailleurs.

$z^{\prime}, z^{\prime \prime}$ : dénivellation du sol te long diune conduite secondaire.

\% : coefficient mumerique (chule do Q).

3 : coefficient mumerique (ehute de $Q$ ).

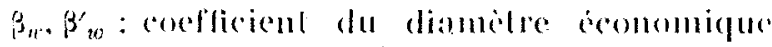
de la conduite de refoulement riminimum de $\mathrm{W}, \mathrm{U})$.

$\gamma:=1.000 \mathrm{~kg} / \mathrm{m}:$, prids sperilique de l'ean.

$د=\left(D_{k-1}-D_{k}\right) /\left(D_{k-\ldots 1}+L_{k}\right)$.

$\delta=4,87$ : indiee de puissance de 1 (perle d'énergie).

$\varepsilon=1,5$ (parfois 1,0$)$ : indice de puissance de $\mathrm{H}$ (prix du réservoir).

$n_{1}=$ rendement du groupe moto-pompe; abscisse numérique.

$\Theta:$ dỉbit numérique.

$\theta$ : nombre d'ammées de remboursement du capital investi.

$\xi$ : abscisse numérique; longueur supplémentaire de la conduite principale.

$\left.\lambda_{,} \lambda_{1}, \lambda^{\prime},\right\rangle^{\prime \prime}$ : paramitres inconnus.

$p, \alpha^{\prime}$ : parametres inconnus.

c: taux de remboursenent du capital,

$\sigma$ : coefficient numérique de la chute du débit.

ๆ. "ૅ : fonctions auxiliaires.

山, : fonctions anxiliaires.

$\Omega$ : débit numérique.

(1) 1,5 : indice de puissance de D (prix des conduites). 


\section{I. - INTRODUCTION}

1. - Dans une localité urbaine ou rurale, l'installation d'une distribution d'eau comprend, en général, les éléments suivants (fig. 1) :
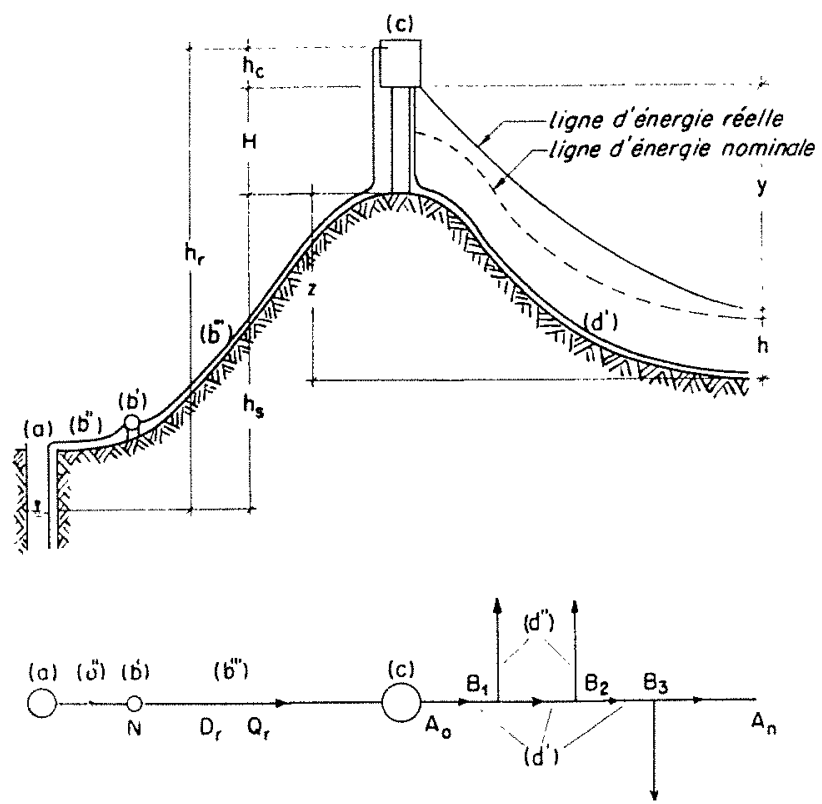

Fig. 1. - Schema d'une distribution d'ean.

a) La prise (cours d'eau, lac, source, puits, forage);

b) La conduite d'adduction amenant l'eau de la prise jusqu'au centre de distribution, soit par gravité (captage surélevé), soit par pompage (captage bas). Une pompe $\left(b^{\prime}\right)$ nécessite une conduite d'aspiration $\left(b^{\prime \prime}\right)$ et une conduite de refoulement $\left(b^{\prime \prime \prime}\right)$;

c) Le centre de distribution (réservoir, château d'eau) où l'eau est emmagasinée, soit pour eréer la charge requise (incendie, douches, in-

\section{I. - INTRODUCTION}

1. In an urban or rural community, the water supply net generally comprises the following elements (fig. 1) :

a) Source or intake of water (water course, lake, spring, well. boring).

b) Supply conduit for the transportation of water from the scurce to the center of distribution, either by gravity or by pumping. A pump $\left(b^{\prime}\right)$ requires a suction conduit $\left(b^{\prime \prime}\right)$ and a delivery conduit $\left(b^{\prime \prime \prime}\right)$.

c) Center of distribulion (rescrvoir, water tower) where water is stored, either in order to produce the required head (fire, shower, irrigation, industry) or to obviate fluctuations in water consumption.

d) Distribution network in the community. It comprises the dustrie, arrosage), soi! pour parer aux fluctuations de la consommation;

d) Le réseau de dislribution dans la localité. Il comprend la conduite principale $\left(d^{\prime}\right)$ sur laquelle se branchent en $B_{1}, \ldots B_{5}$ des conduites secondaires $\left(d^{\prime \prime}\right)$, sur celles-ci des conduites tertiaires, etc., jusqu'aux conduites amenant l'eau chez le consommateur. On distingue ici le réseau ramifié (fig. $2 a$ ) qui est plus simple à calculer, du résea maillé (fig. $2 b$ ) qui est plus pratique el sûr.

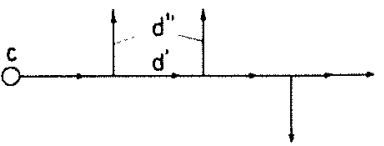

(a)

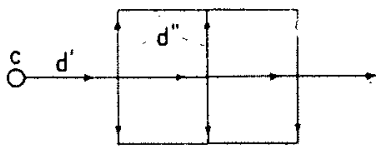

(b)
Fri, 2. -... Schchas de réseaux :

a) ramifiés; b) maillés.

2. - L’ingénieur chargé de préparer les plans d'une installation de distribution d'aulu doit déterminer :

a) Les éléments lopograpiniques en plan el en hauteur; la prise et son niveau; l'emplacement du réservoir; le type du réseau de distribution ef le trace de toutes les conduites, y compris le sens d'écoulement de l'eau;

b) Les éléments hydrauliques dépendant des besoins des consonmateurs : débits maxima $Q$ dans toutes les conduites ef pression minimum $p$ (ou hauteur d'eau équivalente $h$ ) en chaque point du réseau, déterminćs par la densité de la population, des industries et des jardins, et surtout par les dangers d'incendie. Ces grandeurs sont des prédictions basées sur les statistiques du passé et l'état présent, ainsi que sur la comparaison avec des localités semblables. Les débits

main $\left(d^{\prime}\right)$ with submains of order two, branching at $B_{1} \ldots B_{;}$ $\left(d^{\prime \prime}\right)$, submains of order three, etc., up to the conduits leading Io the consumer. We may distinguish the simpler branching nets (fig. 2a) and the complex nets (fig. $2 b$ ) which are more practical and sure.

2. The designing engineer has to determine :

a) The topographical elcments in plan and height; the water intake or source and its level; the reservoir site; the type of the distribution net and the layout of all conduits with the directions of flow.

b) The hydraulic elements depend on the consumer. The maximum discharges $Q$ in all conduits and the minimum pressure $p$ for equivalent pressure head $h$ ) at any point, are determined by the density of population, industry, gardens and fire hazard. These elements are predictions based on past statistics, on present cond- 
dépendent encore des fuites le long des conduites, pertes qui peuvent dépasser $10 \%$ [1] (*). Il faut ajouter à ces éléments le volume du réservoir déterminé par le choix de la réserve d'eau minimum requise par les conditions locales. L'étude économique du réservoir permet d'en déterminer le type est les dimensions intéricures;

c) Les dimensions des éléments hydrauliques du réseau, la hauteur $\mathrm{H}$ du fond du réservoir au-

(*) Les nombres entre crochels se rapportent à la liste bibliographique à la fin du mémoire. Les nombres entre parenthèses se rapportenl aux formules du texte. dessus du sol (fig. 1); ic diamètre $D_{r}$ de la conduite de refoulement ou d'adduction; les diamitres D de tous les tronçons du réseau, telles qu" conduites principales et secondaires; la puissance nominale $\mathbf{N}$, ou installee, du groupe motopompe pour le débit maximum Q refoulé du puits dans le réservoir.

Darss le cas général, ce problème ne peut êre résolu univoquement en termes hydrauliques. En effet, il existe une infinité de solutions (c) satislaisant aux conditions $(a)$ et $(b)$. La solution à choisir est la solution économique qui est unique par définition.

\section{II. - CONSIDERATIONS ÉCONOMIQUES}

3. - La définition de la solution économique nécessite des éclaircissements. On pourrait, par exemple, exiger qu'elle corresponde au minimum de capitaux $\mathrm{W}$ investis dans l'installation. Ce serait la méthode à employer dans le cas d'installations provisoires et militaires, ou dans le cas d'un manque aigu de fonds disponibles. Ces inrestissements sont les suivants :

a) Poste de pompage $W_{1}$,

b) Réservoir d'eau $W_{2}$,

c) Conduite d'adduction $\mathrm{W}_{3}$,

d) Conduite principale $\mathrm{W}_{4}$,

e) Conduites secondaires $W_{5}$,

f) Captage, installations et bâtiments auxiliaires $\mathrm{W}_{6}$,

g) Dépenses initiales, intérêt pendant la póriode de construction, etc., $W_{\tau}$.

itions, and on comparison with similar localities. The discharges are influenced by leakage which may exceed $10 \%$ [I] $\left(^{*}\right)$.

Another element is the reservoir volume determined by lccal requirements of minimum water storage. The economic study of the reservoir determines its type and size.

c) The sizes of the bydraulic elements of the network: height $\mathrm{H}$ of the reservoir bottom above ground level; diameter $D_{\text {, }}$ of the delivery pipe; diameters $D$ of all mains and submains; rated power $N$ of the pumping units for maximum discharge $Q$, delivered into the reservoir.

Generally this problem is hydraulically indeterminate, as an infinite number of solutions ( $c$ ) exist satisfying conditions $(a)$ and (b). The required answer is the economical solution, which is unique by definition.

\section{1. - ECONOMIC CONSIDERATIONS}

3. The economical solution may correspond, for example, to

(*) Numbers within square brackets refer to the bibliography at the end of the paper. Numlers within ortinary brackets refer to the formulite of the text.
Le total des capilaux investis est donc :

$$
\begin{aligned}
\mathrm{W} \equiv \mathrm{I} \mathrm{W}_{j}=\mathrm{W}_{1}+\mathrm{W}_{2_{2}}+\mathrm{W}_{3} \\
\quad+\mathrm{W}_{4}+\mathrm{W}_{5}+\mathrm{W}_{6}+\mathrm{W}_{7}
\end{aligned}
$$

4. - On préfère toutefois, surtout dans le cas d'installations durables, délinir la solution économique par le minimum de dépenses annuelles U. Ces dépenses comprennent: frais de production jusqu'à la station de pompage; traitement de l'eau (filtrage, chlore); transport de l'eau jusqu'au centre de distribution; réseau de distribution, partie publique et partie privée; administration générale, salaires et impôts; pertes d'eau, soit par les fuites le long des conduiles, soit par l'indication erronée de compteurs d'eau usagés. Ces dépenses annuelles rapportées à l'unité de volume $\left(\mathrm{m}^{3}\right)$ d'eau fournic aux consommateurs définissent le prix de l'eall. La solution économique, c'est la recherche du prix minimum.

the minimum of capital investment $W$ in the water supply system. This is recommended for provisional or military installations, or where the necessary funds are not available. These investments comprise : pumping station $\mathrm{W}_{1}$; water reservoir $\mathrm{W}_{2}$; supply (or delivery) conduit $W_{3}$; main $W_{4}$; submains $W_{\pi ;}$; water intake. auxiliary buildings and installations $W_{G ;}$; initial expenditure, capital interest during construction, etc. $W_{7}$.

The total capital investment is : (1)

4. The economical solution is usually defined, especially for lasting installations, by the minimum of annual expenditure $U$. This expenditure comprises: production cost up to the pumping station; water treatment (filters, chlorination); transportation of water to the center of distribution; distribution network, public and private portions; general administration, wages and taxes; water losses by leakage or defective water meters. This expenditure on the basis of unit volume $\left(\mathrm{m}^{3}\right)$ of water supplied to the consumer, defines the water price. The economical solution means the minimum price. 
5. - Les dépenses annuelles peuvent encore ètre disséquées autrement :

a) Intérèt $U_{1}$ sur les capitaux investis $W$ au taux annuel $r$ (par exemple 0,05 , soit $5 \%$ ):

$$
\mathrm{U}_{1}=r \mathrm{~W}
$$

b) Amortissement $\mathrm{U}_{2}$ des diverses parties de l'installation dù à leur détérioration progressive. Si la durée de vie d'un élément $j$ est $\mathrm{T}_{j}$ ans au taux d'intérêt $r$, son taux d'amortissement annuel $r_{j}$ est $\left(^{\star}\right)$ :

$$
\begin{aligned}
& r_{j}=r /\left[(1+r)^{\mathrm{x}_{j}}-1\right] \\
& \mathrm{U}_{2}=\Sigma r_{j} \mathrm{~W}_{j}
\end{aligned}
$$

c) Entretien, réparations et assurance $\mathrm{U}_{3}$ rapportés aux capitaux investis, au taux annuel $p_{j}\left({ }^{\star *}\right)$ :

$$
\mathrm{U}_{3}=\Sigma p_{j} \mathrm{~W}_{j}
$$

d) Pompage $\mathrm{U}_{4}$. Dans le cas d'un moteur électrique, la puissance $\mathrm{N}(\mathrm{kgm} / \mathrm{s})$ est (fig. 1) :

$\mathrm{N}=\gamma \mathrm{Q}_{r}\left(h_{r}+y_{r}\right) / r_{\mathrm{i}}=\gamma \eta^{-1} \mathrm{Q}_{r}\left(h_{s}+h_{c}+\mathrm{H}+y_{r}\right)$

où :

$Q,\left(\mathrm{~m}^{3} / \mathrm{s}\right)=$ débil refoulé maximum;

$h_{r}=$ hauteur de pompage;

$y_{r}=$ perte de charge dans la conduite d'adduction;

$y=1.000 \mathrm{~kg} / \mathrm{m}^{*}$, poids spécifique de l'eau;

$r_{1}=$ rendement du groupe moto-pompe;

$\boldsymbol{H}=$ hauteur du fond au-dessus du sol;

$h_{i}=$ hatuteur de la conduite de refoulement au-dessus du fond.

(*) Les valeurs usuelles de $\mathrm{T}_{j}$ et $r_{j}$ pour $r=0,05$ (soit $5 \%$ ) sont données à la fin du mémoire (Appendice $A$ ).

(*) Les valeurs usuelles de $p_{j}$ sont données à la fiu du mémoire (Appendice $B$ ).

5. Annual expenditure may also be analyzed otherwise :

a) Interest $U_{1}$ on capital investment $W$ at the annual rate $r$ (e.g. 0.05 or $5 \%$ : (2)

b) Amortisation $\mathrm{U}_{2}$ of the various parts of the installation due to their progressive deterioration. If the duration of each element $j$ is $T_{j}$ years at the rate of interest $r$, its annual rate of amortisation $r_{j}(*)$ is : (3) (4)

c) Maintenance, repairs and insurance $\mathrm{U}_{3}$ referred to the capital investments, at an annual rate $p_{j}\left({ }^{*}\right):(5)$

d) Pumping costs $U_{4}$. In the case of an electric motor of power $\mathrm{N}(\mathrm{kgm} / \mathrm{s})$ (fig. 1) : (6)

$\mathrm{Q}_{r}\left(\mathrm{~m}^{3} / \mathrm{s}\right)=$ maximum discharge; $h_{r}=$ pumping height; $y_{r}=$ loss of head in supply conduit; $\gamma=1000 \mathrm{~kg} / \mathrm{m}^{3}=$ unit weight of water; $\eta=$ efficiency of pumping unit; $H=$ height of reservoir bottom above ground; $h_{\epsilon}=$ height of delivery conduit above bottom.

(*) Detailed values of $T_{3}, r_{3}, p_{1}$ are given in Appendixes $A, B$ of the French text.
Lorsque le débit et la hauteur de pompage varient pendant l'annéc, on prend leurs valeurs moyennes annuelles. Si la pompe travaille au cours de l'année pendant un temps $t$ (sec), l'énergie requise est $\mathrm{N} t(\mathrm{kgm})$. Si le prix de l'énergie est $e$ (par $\mathrm{kgm}$, soit $367.000 e$ par $k W h)$, le coût annuel de pompage est :

$$
\mathrm{U}_{4}=\mathrm{N} t e
$$

e) Charges fixes $\mathrm{U}_{5}$, tels salaires, impôts, traitement de l'eau, etc. Les dépenses annuelles totales sont donc :

$\mathrm{U}=\mathrm{\Sigma} \mathrm{U}_{j}=r \mathrm{~W}+\Sigma r_{j} \mathrm{~W}_{j}+\mathrm{v} p_{j} \mathrm{~W}_{j}+\mathrm{N} e t+\mathrm{U}_{5}$

$$
==\mathrm{N}\left(\boldsymbol{r}+r_{j}+p_{j}\right) \mathrm{W}_{j}+\mathrm{Net}+\mathrm{U}_{5}
$$

6. -- Parfois, il laut rembourser les capilaux $W$ pendant 0 années au taux annuel $f$; $r$ étant le taux d'intérèt, on a :

$$
\xi=r /\left[(l+r)^{b--l]}\right.
$$

Il faut ajouter, pendant les ${ }^{9}$ premières annces, une annuité :

$$
\mathrm{U}_{\mathrm{b}}=\rho \mathrm{W}
$$

Les dépenses annuelles deviennent alors :

$$
\mathrm{U}=\mathbf{\Sigma}\left(\boldsymbol{r}+\rho+r_{j}+p_{j}\right) \mathrm{W}_{j}+\mathrm{Ne} t+\mathrm{U}_{j}
$$

7. - Conduites. Le prix d'une conduite est proportionnel à sa longueur $l$ ou $\mathrm{l}$. Le prix par nètre courant est une fonction croissante du diametre intérieur D. On peut le représenter à peu près par une parabole $\left(^{*}\right)$ :

$$
\mathrm{P}=a+b \mathrm{D}^{\mathrm{s}}
$$

( ${ }^{*}$ Sa justification est donnée à la fin du mémoire (Appendice C).

When the discharge and the pumping head vary during the year, they should be replaced by their annual averages. If the pump works $t$ (seconds) a year, the energy required is $\mathrm{N} t(\mathbf{k g m})$. If the price of energy is e (per $\mathrm{kgm}$, or $367,000 \mathrm{e} / \mathrm{kWh}$ ), the annual cost is : $\left(6^{\prime}\right)$

e) Constant expenditure $U_{5}$, such as wages, taxes, water treat. ment, etc.

The total annual expenditure $U$ is then: (7)

6. Sometimes it is necessary to refund the capital $W$ during $\checkmark$ years at an annual rate $0 ; r$ being the annual rate of interest. Then : (8)

During the first 8 years, we have an annual expenditure : (9)

The total annual expenditures are then: $\left(\boldsymbol{g}^{\prime}\right)$

7. Conduits.-The price of a pipe-line is directly proportional to its length $l$ or $L$. The price per unit length (mueter) is an increasing function of the internal diameter $D$. It may be represented approximately by a parabola $\left(^{*}\right):(10)$

(*) See Appendixes C, D of the French text 
où $a, b, \omega$, sont des paramètres à déterminer dans chaque cas $\left({ }^{*}\right)$. En général $\left(^{* *}\right)$ :

$$
1<(1)<2
$$

Dans ce qui suit, nous adopterons $\omega=1,5$.

Le coût de la conduite d'adduction de longueur $L_{r}$ est :

$$
\mathrm{W}_{3}=\mathrm{P}_{r} \mathrm{~L}_{r}=\left(\boldsymbol{a}+\boldsymbol{b} \mathrm{D}_{r}{ }^{\omega}\right) \mathrm{L}_{r}
$$

Le coût de la conduite principale de longueur totale $\mathrm{L}$, composée de $n$ tronçons $l_{k}$ de diamètre $\mathrm{D}_{k}(k=0,1 \ldots n-1)$ est :

$$
\mathrm{W}_{4}=\Sigma \mathrm{P}_{k} l_{k}=\Sigma\left(a+b \mathrm{D}_{k}^{\omega}\right) l_{l} ; \mathrm{L}=\Sigma l_{k}
$$

Le coût des conduites secondaires composées de $n^{\prime}$ tronçons $l_{k}^{\prime}$ de diamètre $\mathrm{D}_{k}^{\prime}(k=0,1 \ldots$, $\left.n^{\prime}-1\right)$ est :

$$
\mathrm{W}_{5}=\Sigma \mathrm{P}_{k i}^{\prime} l_{l i}^{\prime}=\mathrm{N}\left(a+b \mathrm{D}_{k_{k}}^{\prime \omega}\right) l_{k}^{\prime}
$$

8. - Réservoir d'eau. Pour un type et un volume donnés, le coùt $W_{2}$ d'un château d'eatu est fonction de la hauteur $\mathrm{H}$ du fond au-dessus du sol (fig. 1). On peut le représenter par :

$$
W_{2}=a_{2}+b_{2} H^{\prime}
$$

où $a_{2}, b_{2}, \varepsilon$, sont des paramètres dépendant du volune du réservoir et des prix, à déterminer dans chaque cas. Il semble que $\varepsilon=1,5$. Lorsqu'on n'a pas assez de données, on peut admetIre $\varepsilon=1$.

(**) Une méthode semi-graphique pour trouver les paramètres est donnée à la fin du mémoire (Appendice D).

$(* *)$ D'après Davis [4] pour des conduites en fonte avec accessoires et mise en place, $\omega=1,55$. D'après Parkeh $[8], \omega=1,50$. D'après Kinsanoff $[7], \omega=1,7$. l'après l'auteur, pour des conduites de grand diamètre (2) à $5 \mathrm{~m}$ ) en béton précontraint, $\omega=1,5$, les frais d'excavation et de remplissage y compris; 0 est indépendaut de la pression de l'eau et du type de sol [3]. D'après Davis, les frais d'excavation et de remplissage sont proportionnels au diamètre D [4].

$a, b,(1)$ are parameters to be determined in each case.

In general $1<\omega<2$.

In what follows $\omega=1.5$ is adopted.

The cost of the supply pipe of length $L_{r}$ is : (11)

The cost of the main of total length $L$. composed of $n$ sections of length $l_{k}$ and diameter $D_{k}$ each $(k=0,1, \ldots \mathrm{n}-1)$ is : (12)

The cost of submains, composed of $n^{\prime}$ sections $l_{k}$ of diameter $\mathrm{D}_{k}^{\prime}$ each $\left(k=0,1, \ldots \mathrm{n}^{\prime}-1\right)$ is: (13)

8. Water reservoir.-For given type and volume, the cost $W$ of a water tower depends on the height $\mathrm{H}$ of its bottom above ground (fig. 1). It may be represented by : (14)

$a_{2}, b_{2}, \varepsilon$ are parameters to be determined in each case, depending on the prices and the reservoir volume. It seems that $\varepsilon=1.5$. When no sufficient data are available $\varepsilon=1$ may be assumed.

9. Pumping station,-The cost $W_{1}$ of the pumping unit and station depends on the installed power, therefore also on the rated power N. Il seems that: (15)
9.--Poste de pompage. Le cont $\mathrm{W}_{1}$ du groupe moto-pompe avec le poste de pompage est fonction de la puissance installée, donc aussi de la puissance nominale $\mathrm{N}$. Il semble que la relation soit linéaire :

$$
W_{1}=a_{1}+b_{1} N
$$

où $a_{1}, b_{1}$ sont des piramitres à determiner dans chaque cas.

Autres dépenses. Les autres dépenses $W_{6}, W_{7}$ ou $\mathrm{U}_{5}$ (traitement de l'cau, administration, salaires, etc.), ne dépendent pas des diamètres, de la hauteur du réservoir ni de la puissance du moteur.

10. -... Perte de charge dans une conduite. Dans une conduite de diamètre constant D el de longueur $l$, écoulant un débit constanl $Q$ à la vitesse moyenne $b$, on a la relation de continuité :

$$
Q=v \cdot \pi D 2 / 4
$$

et la pente de la ligne d'énergie est:

$$
\mathrm{J}=\eta / l=(f / \mathrm{D})\left(v^{2} / 2\right)
$$

où $y=$ perte d'énergie continue; $v^{2} / 2 g=$ énergie cinétique; $f=$ coefficient de rottement, sans dimensions [6] $\left(^{*}\right)$.

Le coeffieient $f$ est donné par des formules empiriques en fonetion de D el $n$ (ou (Q), I( p) souvent exponentielles :

$$
J=K Q^{n} \mathrm{D} \cdots
$$

où $m$, ò, sont des nombres constants, bien que difrérant un peu selon les auteurs; $K$ dépend encore de la rugositi des parois.

(*) Voir : Appendice $F$, i la fin du mémoire.

$a_{1}, b_{1}$ parameters to be delermined in each case.

Other expenditures $W_{6}, W_{i}, U_{i}$ (water treatment, administration, wages, etc.) do not depend on the diameters, reservoir height or rated power.

10. Loss of head in a conduit.-In a conduit of constant diameter $D$ and length $l$ discharging water at a constant rate of flow $Q$ and mean velocity $v$, continuity requires : (16)

The hydraulic (or energy line) gradient is: (17), where: $y=$ continuous loss of head; $v^{2} / 2 g=$ kinetic energy; $f=$ dimensionless friction coefficient $(*)$;

$f$ is given by empirical formulae as function of $D$ and $v$, (or $Q$, mostly exponential ones: (18), where:

$m$. $\delta$ are constant numbers, differing slightly according to the author. $K$ depends on wall roughness. The most common formulae are:

(*) See Appendix $F$ of the French text. 
Les formules les plus répandues sont:

\begin{tabular}{|c|c|c|c|c|}
\hline $\mathbf{N}^{\circ}$ & Formule de: & $m$ & $\delta$ & Remarques \\
\hline 1 & Chézy-Dupurt & 2 & 5 & ancienne. \\
\hline 2 & ManNing & 2 & 5,33 & $\begin{array}{l}\text { pays de langue } \\
\text { anglaise. }\end{array}$ \\
\hline 3 & ForchHEIMLR & 2 & 5,40 & $\begin{array}{l}\text { pays de langue } \\
\text { allemande. }\end{array}$ \\
\hline 4 & Hazen-Williams ${ }^{*}$ ) & 1,852 & 4,87 & Etats-Unis \\
\hline 5 & BLASIUS-FLAMANT $\left({ }^{\star *}\right)$ & 1,75 & 4,75 & $\begin{array}{l}\text { pays de langue } \\
\text { française. }\end{array}$ \\
\hline
\end{tabular}

La formule de HaZlin-Willians semble occuper une position moyenne, e'est pourquoi nous la préférons.

La perte d'énergie dans une conduite de diamètre constant $D$, de longueur $l$ et de débit $Q$ variable le long de la conduite, est :

$$
\begin{aligned}
& y=\int_{x=-0}^{\cdot l} \mathrm{~J} d x=\mathrm{K} \mathrm{D}^{-5} \int_{r r=0}^{-l} \mathrm{Q}^{m} d x \\
& \mathrm{~K}=10,7 \mathrm{C}^{-m} \text { (unités mètre-seconde). }
\end{aligned}
$$

( $\left.{ }^{\star}\right)$ En unités métriques mixtes : $v(\mathrm{~m} / \mathrm{s}), \mathrm{D}(\mathrm{mm})$, $Q\left(\mathrm{~m}^{3} / \mathrm{h}\right), \mathrm{J}\left(\mathrm{s}_{0}\right)$, on a :

$$
\begin{aligned}
& I=1,131 \times 10^{12}(\mathrm{Q} / \mathrm{C})^{1,852} \mathrm{D}^{-4,87} \\
& v=1,096 \times 10^{-4} \mathrm{CJ} 0,5+\mathrm{D}^{0,63}
\end{aligned}
$$

oú C est un coefficient caractéristique de la rugosité de la paroi. En moyenne $C=100$ pour des tuyaux en fonte après 10 à 15 ans de service. A la fin du memoire (Appendice $b)$ se trouve une table donnant $v$ et $J$ pour $25 \leqslant \mathrm{D} \leqslant 1250 \mathrm{~mm}, 0,2 \leqslant \mathrm{Q} \leqslant 11.000 \mathrm{~m}^{3} / \mathrm{h}$, basée sur les formules précédentes et recalculée par l'auteur en unités métriques pour les besoins de l'enseignement de l'hydraulique à l'Israël Institute of Technology, Haïfa, Israël.

\begin{tabular}{|c|c|c|c|c|}
\hline$N_{o}$ & Formula of & $m$ & $\delta$ & Notes \\
\hline 1 & Chézy-Dupuit & 2 & 5 & ancient \\
\hline 2 & Manning & 2 & 5.33 & $\begin{array}{l}\text { in Anglo-Saxon } \\
\text { countries }\end{array}$ \\
\hline 3 & Forchheimer & 2 & 5.40 & $\begin{array}{l}\text { in German speaking } \\
\text { countries }\end{array}$ \\
\hline 4 & Hazen-Williams & 1.852 & 4.87 & in U.S.A. \\
\hline 5 & Blasius-Flamant & 1.75 & 4.75 & $\begin{array}{l}\text { in French speaking } \\
\text { countries }\end{array}$ \\
\hline
\end{tabular}

(*) C'est la formule rationnelle semi-empirique de l'écoulement lisse, dite des tuyaux lisses. (Voir : Appendice $F$, fin du mémoire.)

We prefer the Hazen-Williams formula, as it occupies a median position.

The loss of head in a conduit of constant diameter $\mathrm{D}$, length $l$
$(\mathrm{K}=0,00212$ pour $\mathrm{C}=100$ )

Pour tenir compte des pertes locales (accessoires, entrée, coudes), il faut multiplier $K$ par un facteur, dont la valeur est 1,15 à 1,20 en moyenne.

11. -.. La conduite d'adduction a un diametre D). el un débit $\mathrm{Q}_{r}$ constants et une longueur $\mathrm{L}_{r}$.

La conduite principale est formée de $n$ troncons $\mathbf{A}_{0} \mathbf{A}_{1}, \ldots \mathbf{A}_{t} \mathbf{A}_{k+1}, \ldots \mathbf{A}_{n-1} \mathbf{A}_{n}$ de diamèires constants $\mathrm{D}_{0}, \ldots \mathrm{D}_{l,}, \ldots D_{n-1}$ et de longueurs $l_{0}, \ldots l_{k}, \ldots l_{n-1}$ respectivement. Les abscisses des nouds $A_{0}, \ldots A_{k}, \ldots A_{n}$ sont 0 $\ldots x_{k}, \ldots x_{n-1}$, L (fig. 3).

Débit

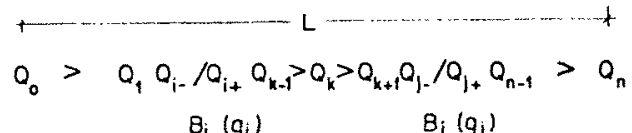

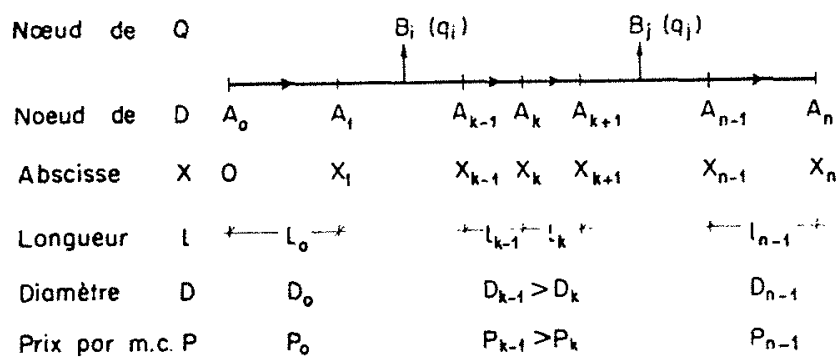

Fiti. 3. - Schéma de la conduite principale.

Le débit décroît le long de la conduite de $Q_{0}$ en $\mathrm{A}_{0}$ à $\mathrm{Q}_{n}$ en $\mathrm{A}_{n}$. Aux nœuds $\mathrm{B}_{i}, \mathrm{~B}_{j}$, des conduites secondaires, il tombe brusquement de $Q_{i-}$ à $Q_{i+}$, etc., $Q_{i-}-Q_{i+}=q_{i}$ représentant le débit de la conduite secondaire $B_{i}$. Ailleurs, il varie d'une facon continue, en assimilant les consommateurs nombreux mais peu importants, et les fuites à une consommation continue $|d Q / d x|$ par mètre courant. La courbe $Q(x)$ a l'allure d'une courbe à gradins (fig. 4$)\left({ }^{*}\right)$.

(*) Pour la rendre plus accessible a l'analyse mathématique, elle sera remplacée par une courbe continue infiniment proche, ce qui est toujours possible et n'a guère d'effet sur le résultat.

and discharge $Q$ varying along the conduit, is : (19)

$K=10.7 \mathrm{Cm}$ (metric units); $\mathrm{K}=0.00212$ for $\mathrm{C}=100$.

Minor losses may be taken into account by multiplying by 1.15 to 1.20 .

11. The delivery conduil has constant diameter $\mathrm{D}_{r}$, discharge

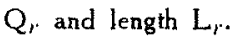

The main is formed of $n$ sections :

$\mathrm{A}_{0} \mathrm{~A}_{1}, \ldots \mathrm{A}_{k} \mathrm{~A}_{k+1}, \ldots \mathrm{A}_{n-1} \mathrm{~A}_{n}$ of constant diameters $\mathrm{D}_{o}, \ldots \mathrm{D}_{k}, \ldots, \mathrm{D}_{n-1}$ and lengths $l_{0}, \ldots l_{k}, \ldots l_{n-1}$ respectively. The abscissae of the branching points $\mathrm{A}_{0}, \ldots \mathrm{A}_{l i}$, $\ldots \mathrm{A}_{n}$ are $0, \ldots x_{k}, \ldots x_{n-1}, \mathrm{~L}$ (fig. 3 ).

The discharge decreases along the conduit from $Q_{1}$, at $A_{0}$ to $Q_{n}$ at $A_{n}$. At the junctions $B_{i}, B_{j}$ of the submains it drops suddenly from $Q_{i-}$ to $Q_{i+}$, etc., $\left[Q_{i-}-Q_{i_{+}}=q_{i}\right]$ representing the discharge through the submain $B_{i}$. Elsewhere it varies continuously, when numerous small consumers and leakage losses are replaced by a continuous consumption $|d Q / d x|$ per unit length. The curve $\mathrm{Q}(x)$ is formed of steps (fig. 4). 


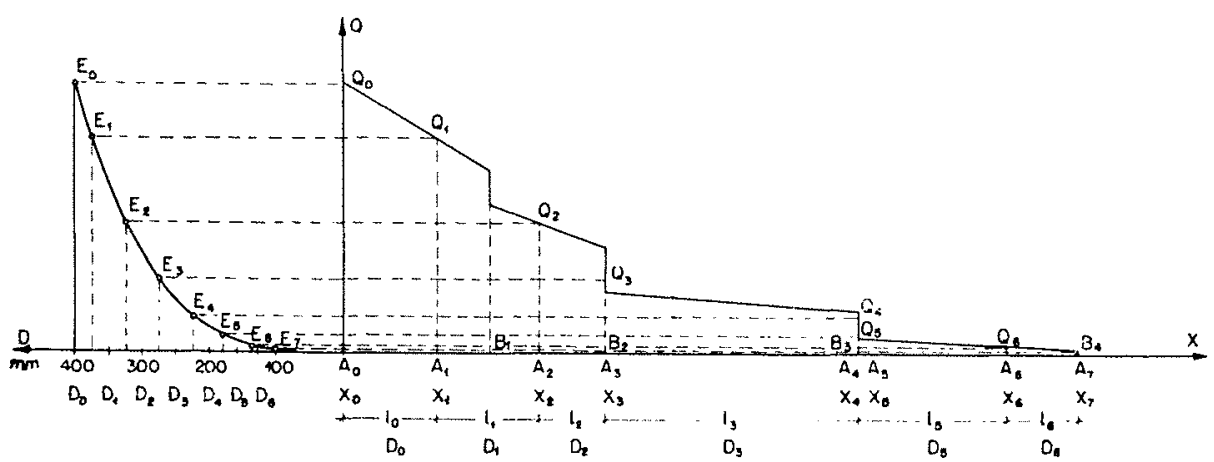

Fig. 4.

Méthode graphique. dite « de la parabole économique * d'une conduite.
12. - Ia solution économique exige l'alternalive :

$\int$ minimum du capilal investi $W$, ou

minimum des dépenses annuelles $\mathrm{U}$.

Il faul y joindre la condition que la perte d'énergie $y$ le long de la conduite principale ne peut dépasser une certaine valeur déterminée par la topographie et les besoins locaux (fig. 1).

$y=\int_{x=0}^{\mathrm{L}} \mathrm{J} d x=\mathrm{K} \int_{x=0}^{\mathrm{L}} \mathrm{Q}^{m} \mathrm{D}-\delta d x \leqslant z+\mathrm{H}-h$ soit :

$$
\psi \equiv y+h-z-\mathbf{H} \leqslant 0
$$

Ici, $\mathrm{W}$ (on $U$ ) est fonction de $(n+2)$ variables:

$x_{k}(k=1,2, \ldots k-1, k, k+1, \ldots n-1)$ est l'abscisse mesurée le long de la conduite développée, en $\mathrm{A}_{k}$ où $\mathrm{D}_{k-1}$ tombe brusquement à $\mathrm{D}_{k}$;

$x_{n}=\mathrm{D}_{\text {r }}$ est le diamètre de la conduite d'adduction;

$x_{n+1}=H$ est la hauteur du château d'eau;

$x_{n+2} \equiv \mathrm{D}_{0}$ est le diamètre inilial de la conduite principale en $A_{0}$.

(*) Au lieu des $x_{k}$ on peut choisir comme variables les $n$ longueurs $l_{k}=x_{t-1}-x_{k}$ des tronçons $A_{k-1} A_{l}$ de diamètre constant $\mathrm{D}_{k}$, chacun.

12. The economical solution requires the alternative : minimum of capital investment $W$, or minimum of annual expenditure $U$.

A further requirement is that the loss of head $y$ along the main cannot exceed a certain value determined by the topography and local needs (fig. 1): (20) or (20')

Here $W$ (or $U$ ) is a function of the $(n+2)$ variables : (21)

The other diameters $D_{k}$ derive from $D_{k}$ by jumps of 10,20 , 25 or $50 \mathrm{~mm}$, according to the producer or availability on the market : $\left(21^{\prime}\right)$
Les autres diametres $\mathrm{D}_{k}(k==1, \ldots n-1)$ découlent de $\mathrm{D}_{0}$ par sauts de $10,20,25$ ou $50 \mathrm{~mm}$, selon la fabrication ou la disponibilite sur le marché local ou international :

$$
\mathrm{D}_{i}=\mathrm{D}_{0}-\text { const. }
$$

el ne sont pas des inconnues indépendantes.

13. - La solution économique conduit is lat recherche du minimum relatif de $\mathrm{W}$ (ou U), fonction de $(n+2)$ variables, ces variables ctant liées par la relation (20). La solution est donnée par la méthode des multiplicateurs de Lacimangs, ce qui revient à rechercher le minimum d'une fonction auxiliaire $F($ ou $G)[5]\left(^{*}\right)$ :

$$
\left\{\begin{array}{l}
F=W+\lambda \psi \\
G=U+\lambda_{1} \psi
\end{array}\right.
$$

$\lambda\left(o u \lambda_{1}\right)$ est un parametre constant, mais inconnu. Les $(n+2)$ inconnues $x_{k}(21)$ et $\lambda$ sont déterminées par les $(n+3)$ équations :

$$
\left\{\begin{array} { r l } 
{ \partial \mathrm { F } / \partial x _ { k } } & { = 0 } \\
{ \psi } & { = 0 }
\end{array} \text { soit } \left\{\begin{array}{rl}
\partial \mathrm{G} / \partial x_{k} & =0(k=1, \ldots n+2) \\
\psi & =0
\end{array}\right.\right.
$$

(*) Lexislence d'un minimum vrai est demontré a la fin du memoire (Appendice G).

and are not independent variables.

13. The economical solution leads towards the study of a relative minimum of $\mathrm{W}$ (or $\mathrm{U})$, function of $(n+2)$ variables. these variables being related by $\left(20^{\prime}\right)$. The solution is given by the method of Lagrangean multipliers, which is equivalent to the research of the minimum of an auxiliary function $F$ (or $\mathrm{G}$ ) [5] $\left.{ }^{*}\right):(22)$, where $\lambda\left(\right.$ or $\left.\lambda_{1}\right)$ is an unknown constant parameter. The $(n+2)$ unknowns $x_{i},(21)$ and $\lambda$ are determined by th: $(n+3)$ equations : (23)

(*) The promif of the existence of a troe minimum is given in Appendix of if the french text. 


\section{III. - CALCUL DES CONDUITES ET MINIMUM DU CAPITAL}

14. - Lexpression explicite de $F$, en remplaçant $W$ par (1), (11) à (15), devient, en négligeant momentanément les conduites secondaires :

$$
\begin{aligned}
\mathbf{F}= & \mathbf{P}_{r} \mathrm{~L}_{r}+\mathrm{W}_{1}+\mathrm{W}_{2}+\lambda(h-z-\mathrm{H})+\int_{x=0}^{x_{1}}\left(\mathrm{P}_{0}+\lambda \mathrm{J}_{0}\right) d x+\ldots \\
& +\int_{a-x_{k-1}}^{x_{k}}\left(\mathrm{P}_{k-1}+\lambda \mathrm{J}_{k-1}\right) d x+\int_{x=k_{k}}^{x_{k+1}}\left(\mathrm{P}_{k}+\lambda \mathrm{J}_{k}\right) d x+\ldots
\end{aligned}
$$

qui est minimum pour :

$$
\begin{aligned}
& \partial \mathrm{F} / \partial x_{k}=\left[\mathrm{P}_{k-1}+\lambda \mathrm{J}_{k-1}\left(x_{i k}\right)\right]-\left[\mathrm{P}_{k}+\lambda \mathrm{J}_{k}\left(\boldsymbol{x}_{k}\right)\right]=0(k=1, \ldots n-1) \\
& \partial \mathrm{F} / \partial x_{n} \equiv \partial \mathrm{F} / \partial \mathrm{D}_{r}=\left(d \mathrm{P}_{r} / d \mathrm{D}_{r}\right) \mathrm{L}_{r}+d \mathrm{~W}_{1} / d \mathrm{D}_{r}=\left(d \mathrm{P}_{r} / d \mathrm{D}_{r}\right) \mathrm{L}_{r}+\left(d \mathrm{~W}_{1} / d \mathrm{~N}\right)\left(\partial \mathrm{N} / \partial \mathrm{D}_{r}\right)=0 \\
& \partial \mathrm{F} / \partial x_{n+1} \equiv \partial \mathrm{F} / \partial \mathrm{H}=d \mathrm{~W}_{2} / d \mathrm{H}-\lambda+\left(d \mathrm{~W}_{1} / d \mathrm{~N}\right)(\partial \mathrm{N} / \partial \mathrm{H})=0 \\
& \partial \mathrm{F} / \partial x_{n+2} \equiv \partial \mathrm{F} / \partial \mathbf{D}_{0}=\sum_{k=0}^{n}\left(\partial \mathrm{F} / \partial \mathrm{D}_{k}\right)\left(d \mathrm{D}_{k} / d \mathrm{D}_{0}\right)=\sum_{n_{i}=0}^{n} \partial \mathrm{F} / \partial \mathrm{D}_{k} \\
& =\sum_{k_{i}}^{n} \int_{x=x_{k}}^{a_{k+1}}\left(\partial \mathrm{P}_{k} / \partial \mathrm{D}_{k}+\lambda \cdot \partial \mathbf{J}_{k} / \partial \mathrm{D}_{k}\right) d x=0
\end{aligned}
$$

en vertu de (21').

15. - Développons (26); en vertu de (6), (10), (18) :

$$
b \omega \mathrm{D}_{r}^{\omega-1} \mathrm{~L}_{r}-b_{1} \gamma \mathrm{Q}_{r} \eta^{-1} \mathrm{~K} \delta \mathrm{Q}^{m} \mathrm{D}_{r}^{-i-1} \mathrm{~L}_{r}=0
$$

soit :

$$
\text { avec : }\left\{\begin{array}{c}
\mathrm{D}_{r}=\beta_{2 n} \mathrm{Q}_{r}{ }^{u} \\
\beta_{1 b}=(\boldsymbol{m}+1) /(\omega+\hat{o})=0,447 \\
=\left(3,24 \mathrm{~K} \gamma / \eta \cdot b_{1} b^{-1} \gamma \eta^{-1} \mathrm{~K}\right)^{1 /(\omega+\delta)}
\end{array}\right.
$$

Le diamètre économique de la conduite d'adduction est indépendant de sa longueur et dépend surtout du débit $\mathrm{Q}_{r}$, presque comme $\mathrm{Q}_{r}{ }^{1 / 2}$. Il dépend un peu de $\eta$ et du rapport $b_{1} / b$ presque à la puissance $1 / 7$.

La pente économique $\mathrm{J}_{r}$ :

$$
\begin{aligned}
\mathrm{J}_{r} & =\mathrm{K} \mathrm{Q}_{r}^{m} \mathrm{D}_{r}^{-\delta} \\
& =\mathrm{K}\left(\omega \hat{o}^{-1} b b_{1}^{-1} \eta \gamma^{-1} \mathrm{~K}\right)^{\delta /(\omega+\delta)} \mathrm{Q}_{r}^{-(n \delta-m)} \\
& =\mathrm{K}^{1,765}\left(0,38 \eta / \gamma \cdot b / b_{1}\right)^{0,7 \pi 5} \mathrm{Q}_{r^{-0,328}}
\end{aligned}
$$

dépend du débit presque comme $\mathrm{Q}_{r^{-1 / 3}}$ et de $r_{i} . b / b_{1}$ presque à la puissance $3 / 4$.

La vilesse économique :

$$
\begin{gathered}
v_{r}=\mathrm{Q}_{r} / 0,785 \mathrm{D}_{r}^{2} \\
=1,275\left(\omega \hat{o}^{-1} b b_{1}^{-1} r_{1} \gamma^{-1} \mathrm{~K}^{-1}\right)^{2 /(\omega+\delta)} \mathrm{Q}_{r^{1-2}} \\
=0,88\left(b / b_{1} \cdot r_{1} / \gamma \mathrm{K}^{\mathrm{n} .314} \mathrm{Q}^{0.116}\right.
\end{gathered}
$$

dépend de $n . b / b_{1}$ presque à la puissance $1 / 3 \mathrm{et}$ fort peu du débit, presque comme $Q_{r}{ }^{1 / 10}$, elle est donc pratiquement constante. Ceci justifie la notion de vitesse économique.

16. - Développons (25); en vertu de (10), (18):

$$
\begin{aligned}
& \lambda \mathbf{Q}_{k}^{m}=\left(\mathbf{P}_{k-1}-\mathbf{P}_{k}\right) /\left(\mathbf{J}_{k}-\mathbf{J}_{k-1}\right)_{s=k} \\
= & \mathrm{K}^{-1}\left(\mathbf{P}_{k-1}-\mathbf{P}_{k}\right)\left(\mathbf{D}_{k}-\delta-\mathbf{D}_{k-1}-\delta\right) \\
= & b \mathrm{~K}^{-1}\left(\mathbf{D}_{k-1} \omega-\mathbf{D}_{k} \omega\right) /\left(\mathbf{D}_{k}-\delta-\mathbf{D}_{k-1}-\delta\right)
\end{aligned}
$$

soit :

$$
\lambda \delta \omega^{-1} \mathrm{~K} b^{-1}=\mathrm{const}=\left(\mathbf{B}_{k} \overline{\mathbf{D}}_{k}\right)^{\kappa+\omega} \mathbf{Q}_{k}^{-m}
$$
où :

\section{III.-COMPUTATION OF CONDUITS AND MINIMUM OF CAPITAL INVESTMENT}

14. Replacing W by (I), (11) to (15), F becomes, when the submains are neglected: (24)

which is minimum for: (25) (26) (27) (28) by virtue of (21').

15. Developping (26) and owing to (6), (10), (18): (29)

The economical diameter of the delivery conduit is independent of its length, and depends mostly on $Q_{r}$, almost as $Q_{r}^{1 / 2}$. It depends slightly on $\eta$ and $b_{1} / b$.

The economical hydraulic gradient $J_{i}:\left(29^{\prime}\right)$ depends on $Q_{\text {r }}$ almost as $\mathrm{Q},{ }^{-1 / 3}$.

The economic velocity $v_{r}:\left(\mathbf{2 9 ^ { \prime \prime }}\right)$ depends very slightly on $Q_{*}$, it is therefore practically constant. This justifies the conception of economical velocity.

16. Developping (25) and owing to (10), (18), we obtain : (30) $\left(30^{\prime}\right)$ or $(31)$.

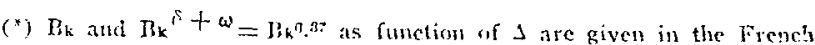
text (pige 145). For $\Delta<0.25$ we have $B_{k}=1-1.06 \Delta^{2}$ within 0.00 : 
$\overline{\mathrm{D}}_{k}=\underset{\text { en } \mathrm{A}_{k} \text {. }}{\left.(\mathrm{I})_{k-1}+\mathrm{D}_{k}\right) / 2 \text { est le diamitre moyen }}$

$\Delta=\left(\mathbf{D}_{k-1}-\mathbf{D}_{k}\right) /\left(\mathbf{D}_{k-1}+\mathbf{D}_{k}\right)$ est l'ćcart relatif des diamètres.

$\mathrm{B}_{k}=\frac{\left[(1+\Delta) \omega-(1-\Delta)^{\omega}\right]}{\left[(1-\Delta)^{-\delta}-(1+\Delta)^{-\delta}\right]}$

$(\omega=1,5 ; \delta=4,87)\left(^{*}\right)$

Au voisinage de $\mathrm{D}_{0}$ (diamètre initial), $\Delta$ est petit, $\mathrm{B} \approx 1$ et $\left(30^{\prime}\right)$ devient :

$\lambda . \omega_{(1)}^{-1} \mathbf{K} b^{-1}=\mathrm{const}$

$$
\begin{aligned}
& =\left(\mathbf{B}_{k i} \overline{\mathbf{D}}_{k}\right)^{\delta+\omega} \mathbf{Q}_{k}^{-m}=\mathbf{D}_{\mathbf{0}^{\delta+\omega}} \mathbf{Q}_{0^{-m}}^{-m} \\
& =\left(\mathrm{B}_{k i} \overline{\mathrm{D}}_{k i}\right)^{\mathbf{6}, 3 \tau} \mathrm{Q}_{k}{ }^{-1,852}=\mathrm{D}_{0}^{6,3 \tau} \mathrm{Q}_{0^{-1,8, \pi}} \\
& \left\{\mathrm{Q}_{k} / \mathrm{Q}_{0}=\left(\mathrm{B}_{k} \overline{\mathrm{D}}_{k} / \mathrm{D}_{0}\right)^{(\delta+\omega) / m}=\left(\mathrm{B}_{k} \overline{\mathrm{D}}_{k} / \mathrm{D}_{0}\right)^{3,4+}\right. \\
& \left\{\mathbf{B}_{k} \overline{\mathbf{D}}_{k} / \mathbf{D}_{0}=\left(\mathbf{Q}_{k} / \mathbf{Q}_{0}\right)^{m /(\delta+\omega)}=\left(\mathbf{Q}_{k} / \mathbf{Q}_{0}\right)^{)^{2,291}}\right.
\end{aligned}
$$

17. - Il en résulte une méthode graphique très simple pour déterminer les abscisses $x_{k}$. On trace (fig. 4) à droite la courbe des débits $Q(x)$ éventuellement rendue continue; et l'on trace à gauche une parabole de degré $(\delta+\omega) / m=3,44$ sur $Q_{0}$ et $D_{0}$ supposé connu $\left.{ }^{* \star}{ }^{\star}\right)$.

Admettons, par exemple, $D_{0}$ connu (soit $400 \mathrm{~mm})$; on trace les diamètres disponibles $\mathrm{D}_{1}(350 \mathrm{~mm}), \mathrm{D}_{2}(300 \mathrm{~mm})$, etc.; puis les diamètres moyens $\overline{\mathrm{D}}_{1}, \overline{\mathrm{D}}_{2}, \ldots$, les écarts relatifs $\Delta$ et les coefficients $B_{k}$. Les valeurs correspondant à notre exemple sont données dans le tableau I cicontre.

Aux abscisses $\left(\mathrm{B}_{k} \overline{\mathrm{D}}_{k}\right)$ on élève des verticales coupant la parabole en $\mathrm{E}_{1}, \mathrm{E}_{2} \ldots$ Des horizon-

\begin{tabular}{|c|c|c|c|c|c|}
\hline$=0$ & 0,05 & 0,10 & 0,15 & 0,20 & 0,25 \\
\hline . & 0,997 & 0,990 & 0,976 & 0,958 &, 935 \\
\hline ,000 & 0,983 & 0,935 & 0,860 & 0,762 & 654 \\
\hline
\end{tabular}
tales menées par ces points coupent la courbe des débits en $Q_{1}, Q_{2} \ldots$ Leurs abscisses $x_{1}, x_{2}, \ldots$

( $\left.{ }^{\star}\right) \mathrm{B}_{k}$ et $\mathrm{B}_{l} \delta+\omega=\mathrm{B}_{k} 6,37$ en fonction de $\Delta$ :

Ln développement en série donne très sensiblement: $\mathrm{B}_{k}=1-1,06 \Delta^{2}$ pour $\Delta<0,25$ (à 0,001 près).

(**) Les ordonnées de cette parabole sont données à la figure 5 .

In the neighborhood of $\mathrm{D}_{0}$ (initial diameter) $\Delta$ is small, $\mathrm{B} \approx 1$ and $\left(30^{\prime}\right)$ becomes : (32)

17. The last formulae lead towards a very simple graphical method for the determination of the abscissae $x_{k}$ where $D_{k-1}$ drops to $D_{k}$. The discharge curve $Q(x)$ (fig. 4) is drawn to the right; to the left a parabola of order $(\delta+\omega) / \mathrm{m}=3.44$ is drawn on $Q_{0}$ and $D_{0}$ supposed known $\left({ }^{*}\right)$.

Let us assume, for example, that $\mathrm{D}_{0}=400 \mathrm{~mm}$. The available diameters $D_{1}(350 \mathrm{~mm}), D_{2}(300 \mathrm{~mm})$, etc., are put down; then the mean diameters $\bar{D}_{1}, \bar{D}_{2}, \ldots$ the relative decrease $\Delta$ and the (**) The ordinates of this parabolia are given in fig. 5 .

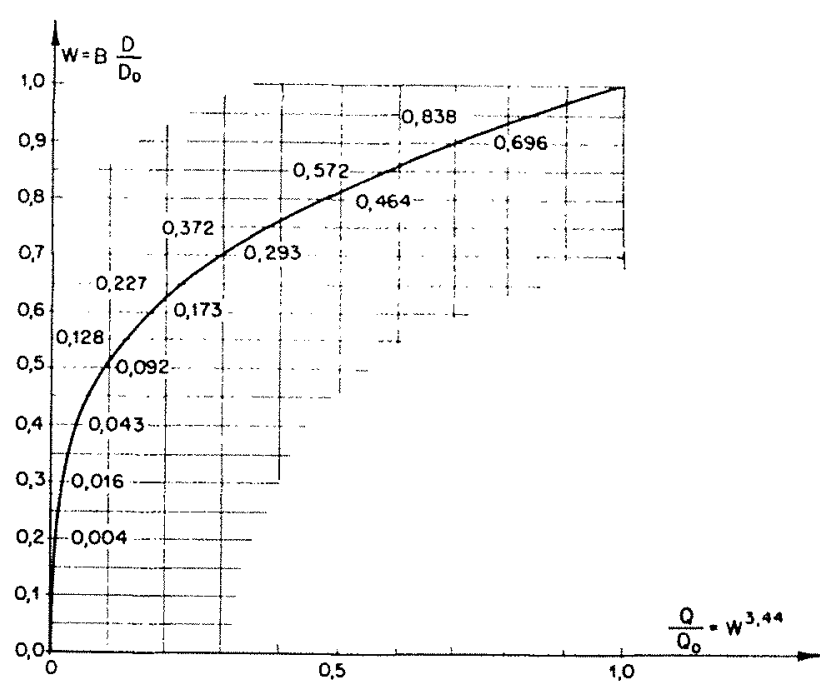

Fici. 5. -- Parabole w3.4.

Tableau I

\begin{tabular}{c|c|c|c|c|c}
\hline$k$ & $\mathrm{D}_{k}$ & $\overline{\mathrm{D}}_{k}$ & $\Delta$ & $\mathrm{B}_{k}$ & $\mathrm{~B}_{k} \overline{\mathrm{D}}_{k}$ \\
\hline 0 & 400 & $(400)$ & & $(1,000)$ & $(400)$ \\
\hline 1 & 350 & 375 & 0,067 & 0,995 & 373 \\
2 & 300 & 325 & 0,077 & 0,993 & 323 \\
3 & 250 & 275 & 0,091 & 0,992 & 273 \\
4 & 200 & 225 & 0,111 & 0,987 & 222 \\
5 & 150 & 175 & 0,143 & 0,980 & 171 \\
6 & 125 & 138 & 0,091 & 0,992 & 137 \\
\hline
\end{tabular}

définissent les points $A_{1}, A_{2}, \ldots$ où l'on passe de $D_{0} \dot{a} D_{1}$, de $D_{1} \dot{a} D_{2}$, etc.

Il arrive parfois que deux abseisses se confondent (ici $x_{1}=x_{3}$ ) au niveau d'un branchement importanl (ici $B_{3}$ ). Le diamitre $)_{4}$ $(=200 \mathrm{~mm})$ n'est pas à employer, et l'on passe directement de $D_{3}(=250)$ à $D_{5}(=150 \mathrm{~mm})$.

coefficients $\mathrm{B}_{k}$. In our example: please refer to French text above: Tableau I.

Verticals are drawn at the abscissae $\left(\mathrm{B}_{k} \overrightarrow{\mathrm{D}_{k}}\right)$ until they meet the parabola at $E_{1}, E_{2}, \ldots$ Horizontals through these points intersect the discharge curve at $Q_{1}, Q_{2} \ldots$ The abscissae $x_{1}, x_{2}, \ldots$ of the intersections define the points $A_{1}, A_{2}, \ldots$ where $D_{0}$ drops to $D_{1}, D_{1}$ to $D_{2}$, etc.

Sometimes two abscissae are equal (here $x_{4}=x_{i j}$ ) where an important submain branches off $\left(B_{3}\right)$. Then the diameter $D_{4}$ $(200 \mathrm{~mm})$ is not used, and $D_{3}(250 \mathrm{~mm})$ drops $10 D_{5}(150 \mathrm{~mm})$. 
A cause de la pelitesse de $\Delta, B_{l} \approx 1$, donc $B_{k i} \overline{\mathbf{D}}_{k} \approx \overline{\mathrm{D}}_{k}$ (voir table ci-dessus). On peul sans grande erreur élever les verticales aux abscisses $\overline{\mathrm{D}}_{k}$ (mi-chemin entre $\mathrm{D}_{k-1}$ ef $\mathrm{D}_{k i}$ ); (32) derient alor's à peu près :

$$
\left\{\begin{array}{l}
\mathrm{Q}_{k} / \mathrm{Q}_{0}=\left(\overline{\mathrm{D}}_{k} / \mathrm{D}_{0}\right)^{(\delta+\omega) / m}=\left(\overline{\mathrm{D}}_{k} / \mathrm{D}_{0}\right)^{3,4 \cdot 1} \\
\overline{\mathrm{D}}_{k} / \mathrm{D}_{t}=\left(\mathrm{Q}_{k} / \mathrm{Q}_{0}\right)^{m /(\delta+\omega)}=\left(\mathrm{Q}_{k} / \mathrm{Q}_{0}\right)^{0,291}
\end{array}\right.
$$

En remplaçant $\mathrm{D}_{k}$ par $\mathrm{D}_{k}$ et en vertu de (16), (18), on a d'une facon approche :

$$
\begin{aligned}
& \left(v_{k} / v_{0}=\left(\mathrm{D}_{k} / \mathrm{D}_{0}\right)^{1.44}=\left(\mathrm{Q}_{k} / \mathrm{Q}_{0}\right)^{0,418}\right.
\end{aligned}
$$

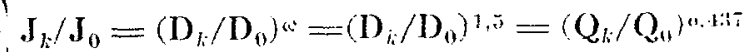

$$
\begin{aligned}
& \text { I }=\left(v_{k} / \nu_{0}\right)^{1, n t}
\end{aligned}
$$

18. - Conclusions.

a) Les vitesses et pentes économiques diminuent le long de la conduite à peu près comme $\mathrm{D}^{1,5}$.

b) Les diamètres économiques décroissent a peu près comme $Q^{0,3}$. Ceei confirme les régles bien connues des praticiens : à petite conduite, petite vitesse; a grosse conduite, grande vitesse; et ligne d'énergie concave vers le haut, c'est-à-dire plus escarpée au début, plus étalée vers la fin [9]. Par contre, il faut rejeter comme contraires à l'économie les conduites à vitesse constante ou à pente d'énergie constante (ligne d'énergie droite) en moyenne.

La méthode graphicque ci-dessus peut s'appeler méthode de la parabole économique d'une conduite de distribution d'eau.

19. - Revenons à (20). Bien que ce soit une inégalité, les solulions précédentes restent valables $\left(^{*}\right)$. On obtient :

$$
y=\sum_{k \rightarrow 0}^{n-1} J_{\mathrm{k}}\left(x_{k}\right) \mathrm{C}_{k_{k}} l_{k} \leqslant z+\mathrm{H}-\boldsymbol{h}
$$

où :

$$
\begin{aligned}
& \mathrm{J}_{k i}\left(x_{i}\right)=\mathrm{K} \mathrm{Q}_{k}^{m} \mathbf{D}_{k^{-5}} ; \\
& C_{k}=\int_{\mathrm{X}=0}^{1} \Omega^{m} d \mathrm{X} ; \Omega(\mathrm{X})=\mathrm{Q}(x) / \mathrm{Q}_{k} \\
& \mathrm{X}=\left(x-x_{k}\right) / l_{k} \quad(0 \leqslant \mathrm{X} \leqslant 1)
\end{aligned}
$$

Le plus souvent, $\Omega$ est une fonction linéaire décroissante du type:

$$
\Omega(\mathrm{X})=l-\beta \mathrm{X} ; \beta=\left(\mathrm{Q}_{k-1}-\mathrm{Q}_{k}\right) / \mathrm{Q}_{k}
$$
donc :

$$
\begin{aligned}
C_{k} & =\left[1-(1-\beta)^{m+1}\right] / \beta(m+1) \\
& =0,351 / \beta \cdot\left[1-(1-\beta)^{2,8,2}\right]\left(^{\star \star}\right)
\end{aligned}
$$

Aı lieu de (34), on peut aussi écrire :

$$
y={\underset{k=0}{\mathrm{D}}}_{\mathrm{\Sigma}}^{n-1} \widehat{J}_{k} \mathrm{G}_{k} l_{k} \leqslant z+\mathrm{H}-\boldsymbol{h}
$$

oì :

\begin{tabular}{|c|c|c|c|c|c|c|c|c|c|c|c|}
\hline$\alpha, \beta=$ & 0,0 & 0,1 & 0,2 & 0,3 & 0,4 & 0,5 & 0,6 & 0,7 & 0,8 & 0,9 & 1,0 \\
\hline $\mathrm{C}_{l_{k}}=$ & 1,000 & 0,910 & 0,826 & 0,746 & 0,673 & 0,604 & 0,541 & 0,484 & 0,433 & 0,386 & 0,347 \\
\hline $\mathrm{G}_{k}=$ & 1,000 & 1,003 & 1,011 & 1,024 & 1,042 & 1,066 & 1,095 & 1,129 & 1,169 & 1,213 & 1,263 \\
\hline
\end{tabular}

$$
\begin{aligned}
& \mathrm{J}_{k}=\mathrm{K} \overline{\mathbf{Q}}_{k}^{-n} \mathbf{D}_{k}^{-\hat{n}} ; \quad \overline{\mathrm{Q}}_{k}=\left(\mathrm{Q}_{k}+\mathrm{Q}_{k+1}\right) / \mathbf{2} ; \\
& \mathrm{G}_{k}=\int_{\xi=0}^{1} \Theta d \xi ; \quad \Theta(\xi)=\mathrm{Q} / \mathrm{Q}_{k} ; \\
& \xi=\left[x-\left(x_{h}+0,5 l_{k}\right)\right] / 0,5 l_{k} ; \quad-1 \leqslant \xi \leqslant 1
\end{aligned}
$$

Le plus souvent, $\Theta$ est une fonction linéaire décroissante telle que $\Theta \equiv 0$ selon que $\xi \leqq 0$, du type :

$$
\Theta=1-\alpha \xi ; \quad x=\left(\mathrm{Q}_{k}-\mathrm{Q}_{k+1}\right) /\left(\mathrm{Q}_{k}+\mathrm{Q}_{k+1}\right)
$$

(*) Il suffit d'écrive $\psi^{*}=y+h-\mathrm{H}-z=-\mathrm{E}^{2}$

$\left(\mathrm{E}^{2}>0\right)$ pour que l'inégalité devienne une égalité.

(“) La table qui suit donne les valeurs de $C_{2 i}(\beta)$ et $\left(x_{k i}(\alpha)\right.$.

En développant les binômes en serrie, on a :

$$
\begin{aligned}
& \mathrm{C}_{k}=1-0,926 \beta+0,263 \beta^{2} \text { à } 1 \% \text { près } \\
& \mathrm{G}_{k}=1+0,263 \alpha^{2}
\end{aligned}
$$

Because of the smallness of $\Delta, \mathrm{B}_{k} \approx 1$, so $\dot{\mathrm{B}}_{k} \overline{\mathrm{D}}_{k} \approx \overline{\mathrm{D}}_{k}$.

Without great error the verticals may be drawn at $\bar{D}_{k}$, half way between $D_{k-1}$ and $D_{k}$. (32) becomes approximately: (32')

Replacing $\widetilde{D}_{k}$ by $D_{k}$, and because of $(16),(18)$, approxim. ately : (33)

18. Conclusions.

a) The economical velocities and hydraulic gradients decrease along the conduits approximately as $D$ i.i.

b) The economical diameters decrease approximately as $Q^{0.2}$.
This confirms the well-known practice : small velocity in small conduit, great velocity in large conduit. Also the energy line is concave upwards : steeper initially, flatter finally [9]. Conduits of constant velocity or of constant hydraulic gradient (straight energy line) are to be rejected as uneconomical.

The above graphical method may be called method of the ccononical parabola of a water supply conduit.

19. 20. Please refer to the French text.

$\ldots D_{o}$ is computed as for a conduit of length $L$ having a constant discharge equal to the initial discharge $Q_{0}$.

This method is simple, quick and sufficiently approximate. 
done :

$$
\begin{aligned}
& \mathrm{G}_{l:}=\left[(1+x)^{m+1} \ldots(1-x)^{m+1}\right] / 2 x(m+1)
\end{aligned}
$$

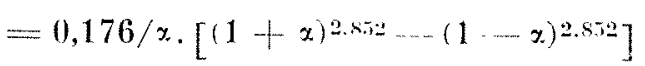

On a tris sensiblement $\mathrm{G}_{k} \approx 1$, done :

$$
y \approx \Sigma \overline{\mathbf{J}}_{k} l_{k} \leqslant z+\mathrm{H}-\boldsymbol{h}
$$

où $\widehat{J}_{k}$ est la pente correspondant à $Q_{k}$, valeur moyenne de $Q$ le long du tronçon $l_{k}$. L'inégalité (34), (36), (38), doit être vérifiće, ce qui sert de contrôle à la valeur admise de $\mathrm{D}_{0}$ et $\mathrm{H}$ qui en résulte.

20. - Afin de rendre les calculs précédents plus simples, on peut se servir de l'approximattion suivante, en partant de (32'), (33):

$$
\begin{aligned}
y & \left.=\mathrm{K} \int_{x=0}^{\mathrm{L}} \mathrm{Q}^{m} \mathrm{D}\right)^{-\delta} d x \\
& =\mathrm{K} \mathrm{Q}_{0}^{m} \mathrm{D}_{0}{ }^{-\delta} \mathrm{L} \quad \int_{\eta=0}^{\mathrm{I}} \mathrm{Q}^{m}\left(\mathrm{D} / \mathrm{D}_{0}\right)^{-\delta} d r_{1} \\
& =\mathrm{J}_{0} \mathrm{R} \mathrm{L} \leqslant z+\mathrm{H}-h
\end{aligned}
$$

où :

$$
\begin{gathered}
\Omega\left(r_{1}\right)=\mathrm{Q} / \mathrm{Q}_{0} ; \quad r_{1}=x / \mathrm{L}\left(^{*}\right) ; \\
\mathrm{R}=\int_{\eta=0}^{1} \Omega^{m \omega /(s+\omega)} d r_{i}=\int_{\eta=0}^{1} \Omega^{0,437} d r_{i}
\end{gathered}
$$

$\Omega\left(n_{i}\right)$ représente, à l'échelle près, $Q(x)$ le long de la conduite principale $\mathrm{L}$.

Cette relation ressemble souvent, en moyenne,

à une parabole, mème lorsque $Q(x)$ est tue courbe à gradins :

$$
\Delta\left(r_{i}\right)=\left(1-\sigma r_{1}\right)^{n}
$$

où $\sigma, n$, sont des coefficients numériques approprics. On trouve $\left.{ }^{\star *}\right)$ :

$$
\begin{aligned}
\mathrm{R} & =\frac{\left[1-(1-\sigma)^{1+m n \omega /(\delta+\omega)}\right]}{\sigma[1+m n \omega /(\hat{\sigma}+\omega)]} \quad\left(40^{\prime}\right) \\
& =[1-(1-\sigma) 1+0.437 n] \sigma(1+0.437 n)
\end{aligned}
$$

Comme $R<1$ ef ne varie que forl lentement avec la forme de la courbe $\Omega\left(x_{i}\right)$ ou $\mathrm{Q}(x)$, on peul tirer de la table une valeur approché de R el l'introduire dans (39), d'ou :

$$
\mathrm{J}_{0} \mathrm{~L}=y / \mathrm{R}>y
$$

Ceci revient is ealculer $D_{1}$ enme pour une conduite de longueur $\mathrm{L}$ et de diamelre constant, mais de perte d'énergie $y / R>y$. Lorsque $R=1$, on a $J_{6} L \approx y$ et $D_{0}$ se calcule comme pour une conduite de longueur I. cooulant un débit conslant égal au débit intial $Q_{0}$. Cette méthode est simple, rapide el suffisamment approchéc.

La solution exacte est (28) qui, développée, donne :

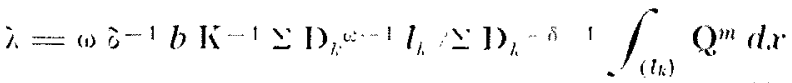

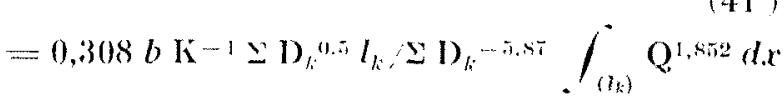

(*) Ne pas confoudre avec le rendement $\eta$ du groupe moto-pompe.

(*) $n=0$ donne une horizontale (débit constant); $n=1$ donne une droile descendante; n< donue une courbe convexe vers le bas et $n>1$ convexe vers le haut. L'n développement en série donne $R=1 \ldots 0,219 n \sigma \ldots .$. La table qui suit donne les valeurs de $R$ en fonction de $\sigma$ et $n$ :

$\begin{array}{llrllllllllll}n & 1+0,437 n & \sigma=0,0 & 0,1 & 0,2 & 0,3 & 0,4 & 0,5 & 0,6 & 0,7 & 0,8 & 0,9 & 1,0 \\ 0,43 & 1,250 & \mathrm{R}=1,00 & 0,98 & 0,97 & 0,96 & 0,94 & 0,93 & 0,91 & 0,89 & 0,87 & 0,84 & 0,80 \\ 1,00 & 1,437 & 1,00 & 0,98 & 0,96 & 0,94 & 0,91 & 0,88 & 0,85 & 0,82 & 0,78 & 0,74 & 0,70 \\ 2,29 & 2,000 & 1,00 & 0,95 & 0,90 & 0,85 & 0,80 & 0,75 & 0,70 & 0,65 & 0,60 & 0,55 & 0,50\end{array}$

\section{IV. - CAlCUL ÉCONOMIQUE DE LA HAUTEUR DU RESERVOIR}

21. -.. Développons (27); en vertu de (14):

$$
\lambda=b_{22} \approx \mathrm{H}^{c-1}+b_{1} \gamma_{r_{i}}^{-1} \mathrm{Q}
$$

Lorsque $\varepsilon=1$ (ou $W_{2}$ est fonction linéaire de $H$, ee qui arrive quand on fait passer la courbe $W_{2}(H)$ par deux points seulement), $\left(30^{\prime \prime}\right)$, (18), (39) donnent:

\section{IV.-COMPUTATION OF ECONOMICAL RESERVOIR HEIGHT}

21. Developing (27) in view of (14), we have: (42) when $\varepsilon=1\left(W_{2}\right.$ is linear in $H$, or $W_{2}(H)$ passes through two points only), (30'), (I8), (39) give: (43)

$$
\begin{aligned}
& \lambda=b_{22}+b_{1} \gamma r_{1}^{-1} \mathrm{Q}_{r}=\omega^{--1} b \mathrm{~K}^{-1} \mathrm{~J}_{0}{ }^{i+1} \omega^{2} \mathrm{Q}_{0}^{-m} \\
& \left.=0,308 b^{-1} \mathrm{~J}_{0}\right)^{6,37} \mathrm{Q}_{0} \cdots 1,852 \\
& \mathrm{D}_{0}=\left(\delta \omega^{-1} \mathrm{~K} \cdot \lambda / b\right)^{1 /(\delta+\omega)} \mathrm{Q}_{0}{ }^{m /(\delta+\omega)} \\
& =(3,24 \mathrm{~K} \cdot 3 / 6)^{0,157} \mathrm{Q}, 6^{0,251}
\end{aligned}
$$

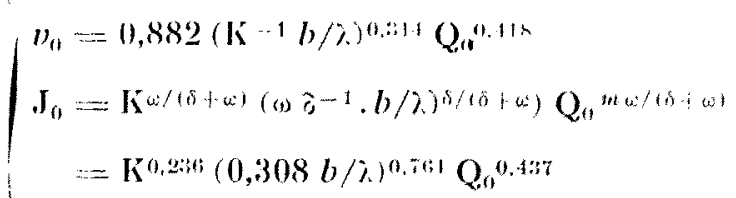

$$
\begin{aligned}
& \mathrm{H} \geqslant \mathrm{J}_{11} \mathrm{RI}+h \cdots z
\end{aligned}
$$


22. - Lorsque $\varepsilon>1$, on trouve, en vertu de (42), $\left(30^{\prime \prime}\right),(39)$ :

$$
\left\{\begin{array}{r}
\mathrm{D}_{0} \leqslant\left[\left(\mathrm{H}^{\varepsilon-1}+b_{1} / b_{2} \cdot \% / \varepsilon \eta \cdot \mathrm{Q}_{r}\right)\right. \\
\left.(\mathrm{H}-h+z) \varepsilon \delta / \omega \cdot b_{2} / b_{1} \cdot 1 / \mathrm{RL}\right]^{1 / \omega} \\
\mathrm{Q}_{0} \leqslant\left[\omega^{-1} b \mathrm{~K}^{-1} /\left(b_{2}+b_{1} \gamma \eta^{-1} \mathrm{Q}_{r}\right)\right]^{1 / m} \\
\mathrm{D}_{0}{ }^{(\delta+\omega) / m}
\end{array}\right.
$$

Ces expressions encombrées sont faciles à calculer et donnent des résultats qualitatifs semblables au cas $\varepsilon=1$, c'est-à-dire : la hauteur du réservoir croît linéairement comme la longueur $\mathrm{L}$ de la conduite; presque comme $Q_{0}{ }^{1 / 2}$ et presque comme :

$$
\left[b /\left(b_{2}+b_{1} \gamma \eta^{-1} Q_{r}\right)\right]^{3 / 4}
$$

23. - Jusqu'ici, nous avons admis que l'emplacement du réservoir est donné à l'avance. Mais parfois, surtout au flane d'un côteau, la question se pose d'éloigner le réservoir si la rěduction de sa hauteur [coût donné par (14)] compense le coût de la longueur $\xi$ à ajouter à la conduite principale $D_{0}$ (coût $P_{0}$ par mètre linéaire).

Soit (fig. 6) un réservoir en $A_{0}$ de hauteur $H_{0}$ calculée économiquement comme ci-dessus. Déplaçons-le de $\xi$ en $A$ où sa hauteur peut être $\mathrm{H}<\mathrm{H}_{0}$

$$
j=z / \xi
$$

est la pente du sol. $J_{0}$ est la pente piézométrique. On a :

$$
\mathrm{H}=\mathrm{H}_{0}+\mathrm{J}_{0} \xi-z=\mathrm{H}_{0}-\left(j-\mathrm{J}_{0}\right) \xi
$$

Comme $0 \leqslant \mathrm{H} \leqslant \mathrm{H}_{0}$, il faut encore que :

$$
\begin{aligned}
& \mathrm{J}_{0} \xi+\mathrm{H}_{0} \geqslant z \geqslant \mathrm{~J}_{0} \xi \\
& \mathrm{J}_{0}+\mathrm{H}_{0} / \xi \geqslant j \geqslant \mathrm{~J}_{0}
\end{aligned}
$$

La condition d'économie exige, si l'on veut éloigner lc réservoir :

$$
\Delta W=\left(a_{2}+b_{2} \mathrm{H}^{c}\right)+\mathrm{P}_{0} \xi<\left(\alpha_{2}+b_{2} \mathrm{H}_{0}\right)
$$

22. Please refer to the French lext.

23. Heretofore the reservoir site was given. Sometimes, especially on a slope, the reservoir is removed. uphill. Its decrease in height [cost (14] compensates for the cost of the additional length $\xi$ of main conduit ( $\mathrm{D}_{0}$; price $\mathrm{P}_{o}$ per unit length).

Let (fig. 6) $A_{o}$ be the reservoir of economical height $H_{o}$ as computed above, and let it move by $\xi$ to $A$, where its height is $\mathrm{H}<\mathrm{H}_{0}$;

$$
j=z / \xi
$$

is the mean surface slope. If $J_{0}$ is the hydraulic gradient, we

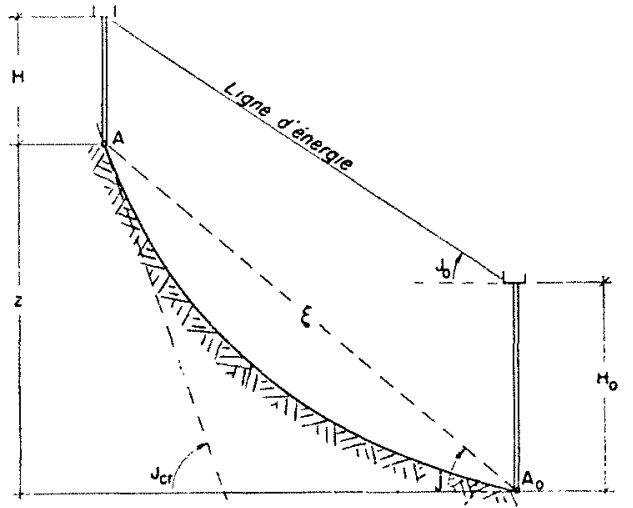

FIti. 6. - Réservoir à flanc de coteau $d z / d \xi=\mathrm{J}_{c r}$.

soit :

$$
\mathrm{H}_{0}^{\varepsilon}-\mathrm{H}^{\varepsilon}>\mathrm{P}_{0} \xi / b_{2}
$$

en négligeant l'effet de $\mathrm{H}$ sur $\mathrm{N}$ (autrement il faut ajouter $b_{1} \gamma \eta^{-1} Q_{r} \mathrm{H}$ et $b_{1} \gamma \eta^{-1} \mathrm{Q}_{r} \mathrm{H}_{0}$ aux deux membres de l'inégalité). En vertu de (46) il faut que :

$$
\begin{aligned}
& j=\mathrm{J}_{0}+\left(\mathrm{H}_{0}-\mathrm{H}\right) / \xi \\
& \quad>\mathrm{J}_{0}+\left[\mathrm{H}_{0}-\left(\mathrm{H}_{0}{ }^{\varepsilon}-\mathrm{P}_{0} \xi / b_{2}\right)^{1 / \varepsilon}\right] / \xi \\
& =\mathrm{J}_{0}+\left[1-\left(1-\mathrm{P}_{0} / b_{2} \cdot \xi / \mathrm{H}_{0}\right)^{1 / \varepsilon}\right] \cdot \mathrm{H}_{0} / \xi \\
& >\mathrm{J}_{0}+\left[1-\left(1-\mathrm{P}_{0} / b_{2} \cdot \xi / \mathrm{H}_{0} \varepsilon\right)^{1 / \varepsilon}\right] \mathrm{P}_{0} / b_{2} \cdot \mathrm{H}_{0}^{\varepsilon-1}
\end{aligned}
$$

c'est-à-dire, il faut que la pente moyenne du sol $j$ soit supérieure à la pente piézométrique $J_{0}$. Mais cette condition ne suffit pas. En effet, lorsque $\varepsilon=1$ (cas simple), il faut que $j$ soit supérieur à la pente économique critique $J_{c r}$.

$$
\mathbf{J}_{c r}=\mathbf{J}_{0}+\mathbf{P}_{0} / b_{2}
$$

ou plus exactement :

$$
J_{e r}=\mathbf{J}_{0}+P_{0} /\left(b_{2}+b_{1} \gamma / \eta \cdot Q_{r}\right)
$$

have : (46)

As $\mathrm{O} \leqslant \mathrm{H} \leqslant \mathrm{H}_{0}$ we require also: $\mathrm{J}_{0} \xi+\mathrm{H}_{0} \geqslant z \geqslant J_{0} \xi$, and : (47) (48)

or

$$
\mathrm{H}_{0}{ }^{\varepsilon}-\mathrm{H} c>\mathrm{P}_{0} \xi / b_{2}
$$

neglecting the effect of $\mathrm{H}$ on $\mathrm{N}$.

The mean surface slope $j$ should be greater than the hydraulic gradient $J_{0}$. This condition does not suffice. When $\varepsilon=1$, for example, $j$ should be greater than the crilical economical slope $J_{C r}:(50)$, or more exactly : $\left(50^{\prime}\right)$ when the effect of $\mathrm{H}$ on $N$ is considered. 
Le minimum de $\Delta W$ a lieu alors pour :

$$
d z / d \xi=J_{c r}
$$

donc là où le sol a la pente critique.

Lorsqu'on a partout $d z / d \xi<\mathrm{J}_{c, r}$, il faut éloigner le réservoir jusqu'à hauteur nulle $(\mathrm{H}=0$ ) pour $\xi=\left(z-\mathrm{H}_{0}\right) / \mathbf{J}_{0}$. L'emplacement $\mathrm{A}$ peut alors être déterminé graphiquement (fig. 7). On mène par le sommet $\mathrm{H}_{0}$ une horizontale $\mathrm{H}_{0} \mathrm{O}$ et par $O$ une droite $O B$ de pente $J_{n}$. On cherche sur elle un point $\mathrm{B}$ tel que $\mathrm{BA}=\mathrm{OH}_{0}=c$. De là, $\mathrm{il}$ découle que :

$$
\mathrm{FA}=\mathrm{OD}=\left(z-\mathrm{H}_{0}\right) / \mathrm{J}_{0}=\xi
$$

A est l'emplacement cherché (nous admettons ici $A F \approx A_{0} A=\xi$.

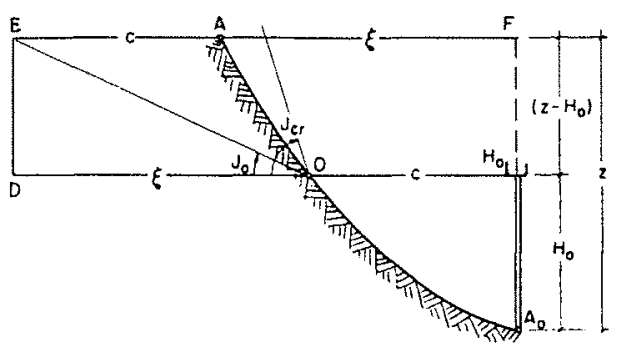

Fla. 7 . - Réservoir à flanc de coteau $j<J_{C r}$.

24. - D'après la topographie, quatre cas sont à considérer selon $I_{a}$ position relative de la ligne d'énergie réelle et de la ligne d'énergie nominale (qui, à l'énergie cinćtique près, se confond avec la ligne de charge nominale, parallcle à la surface du sol à une distance $h$ (fig. 8).

a) Sol concave, ligne d'énergie réelle au-dessus de la ligne nominale. $H$ étant donné, on trouve $y$. Il reste à déterminer tous les diamètres;

b) Sol horizontal, ascendant ou descendant peu. Ici, il faut calculer $\mathrm{H}$ économique;

c) Sol convexe. Puisque Ia ligne d'énergie économique est concave vers le haut, elle coupe la

The minimum of $\Delta \mathrm{W}$ is oblained where the soil is at the critical slope : (51)

When everywhere $d z / d \xi<\mathrm{J}_{\text {r, }}$, the reservoir is to be sited where $\mathrm{H}=\mathrm{O}$ or $\xi=\left(z+\mathrm{H}_{\theta}\right) / \mathrm{J}_{0}$. This site may be found graphically (fig. 7). Through $\mathrm{H}_{o}$ a horizontal $\mathrm{H}_{0} \mathrm{O}$ and through $\mathrm{O}$ a straight line $O B$ of slope $J_{0}$ are drawn. The point $B$ is found where $\mathrm{BA}=\mathrm{OH}_{0}=c$. Thence :

$$
\mathrm{FA}=\mathrm{OD}=\left(\mathrm{z}-\mathrm{H}_{0}\right) / \mathrm{J}_{0}=\xi
$$

We assume $\mathrm{AF} \approx \mathrm{A}_{0} \mathrm{~A}=\xi$.

24. According to the topography, we may consider four cases of the relative position of the true energy line and the rated energy line (parallel to soil surface at the distance $h$ ) (fig. 8).

a) Concave ground : the true energy line is above the rated line.

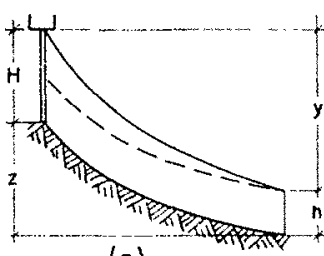

(o)

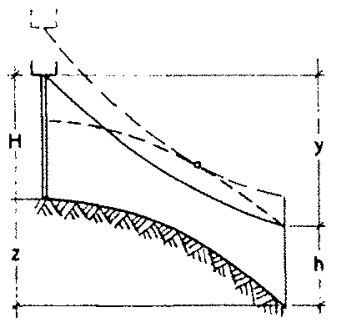

(c)

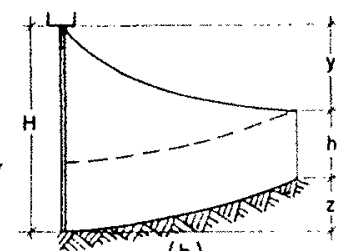

(b)

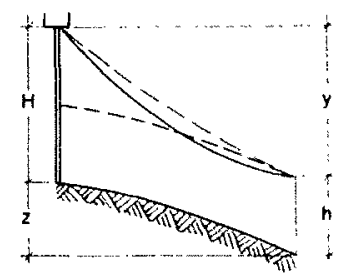

(c')

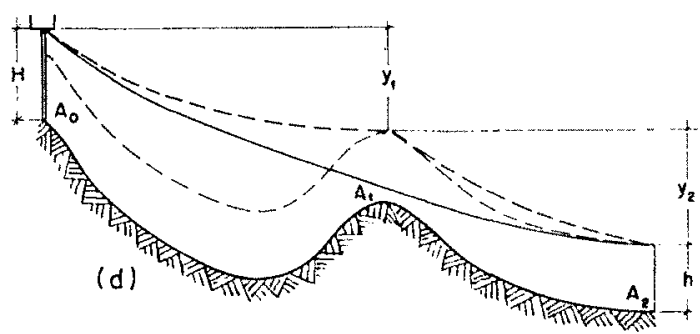

Fro. 8. - Diverses dispositions topographigues : a) concave; b) ascendant; $c$ ) convexe (H aceru); $c^{\prime}$ ) convexe $\left(\mathrm{D}_{0}\right.$ aceru) ; d) onduleux.

ligne d'inergie nominale, qui est convexe. Il f'sut soit conserver $D_{0}$ et augmenter $H$ en déplacint la ligne d'énergie vers le haut jusqu'à ce qu'elle soit tangente à la ligne nominale; soit conserver $H$, mais augmenter $D_{0}$, la ligne d'énergie s'aplatissant jusqu'à devenir tangente à la ligne nominale;

d) Sol onduleux. On trace la ligne d'énergie et on examine ses intersections avec la ligne nominale : on élève la ligne d'énergie jusqu'à ce qu'elle soit tangente à la ligne nominale: on commence a calculer par le bas : d'ahord tronçon (2-1), puis (1-0), finalement $H$.

For a given $\mathrm{H}, y$ is found. The diameters are to be found.

b) Horizontal, rising or slightly descending ground. H economical is to be found.

c) Convex ground. As the economical energy line is concave upwards, it intersects the rated convex line. Either $\mathrm{D}_{0}$, is maintained with $\mathrm{H}$ increased upwards until the energy line is tangent to the rated line; or $\mathrm{H}_{0}$ is maintained, and $\mathrm{D}_{n}$ increased, the energy line flattening out until it becomes tangent to the rated line.

d) Undulating ground. The energy line is examined as to its intersections with the rated line. The energy line is raised until it is tangent to the rated line. Starting from the bottom: first section (2-1), then (1-0), finally $\mathrm{H}$ are computed. 


\section{V. - EFFET DES CONDUITES SECONDAIRES}

25. - Réseal ramifié. Jusquici, nous avons négligé les conduites secondaires. Lorsqu'on en tient compte, on obtient une relation nouvelle pour chaque noud.

Soit une conduite principale A $\mathrm{E} \mathrm{A}^{\prime}$ avec le branchement $E A^{\prime \prime}$ en $E$ (fig. 9).
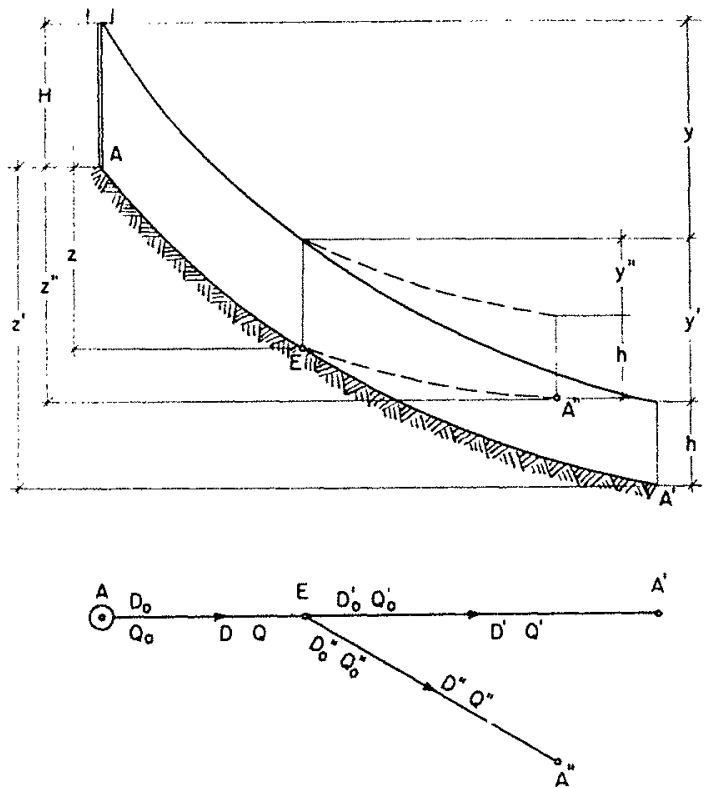

Fici. 9. - Réseau ramifié.

\begin{tabular}{|c|c|c|c|c|c|}
\hline \multirow[b]{2}{*}{ Tronçon } & \multicolumn{2}{|c|}{$!$} & \multicolumn{2}{|c|}{ I } & \multirow[b]{2}{*}{ Pente } \\
\hline & $\begin{array}{l}\text { Diamètre } \\
\text { (variable) }\end{array}$ & $\begin{array}{c}\text { Débit } \\
\text { (variable) }\end{array}$ & Longueur & Perte & \\
\hline$A E$ & $\mathrm{D}$ & $Q$ & L & $y$ & $\mathrm{~J}$ \\
\hline $\mathrm{EA}^{\prime}$ & $D^{\prime}$ & $Q^{\prime}$ & $\mathrm{I}^{\prime}$ & $y^{\prime}$ & $J^{\prime}$ \\
\hline $\mathbf{E} A^{\prime \prime}$ & $D^{\prime \prime}$ & $Q^{\prime \prime}$ & $\mathrm{I}^{\prime \prime}$ & $y^{\prime \prime}$ & $J^{\prime \prime}$ \\
\hline
\end{tabular}

\section{V.-THE EFFECT OF SUBMAINS}

25. Branched network.-When the cost of submains is not neglected, we get a new relation per each junction. Let $\mathrm{AEA}^{\prime}$ (fig. 9) be the main with a submrain $\mathrm{EA}^{\prime \prime}$ branching off at $\mathrm{E}$.

\begin{tabular}{c|c|c|c|c|c}
\hline Section & $\begin{array}{c}\text { Diameter } \\
\text { (variable) }\end{array}$ & $\begin{array}{c}\text { Discharge } \\
\text { (variable) }\end{array}$ & Length & $\begin{array}{c}\text { Loss } \\
\text { of Head }\end{array}$ & Gradient \\
\hline $\mathrm{AE}$ & $\mathrm{D}$ & $\mathrm{Q}$ & $\mathrm{L}$ & $y$ & $\mathrm{~J}$ \\
$\mathrm{EA}^{\prime}$ & $\mathrm{D}^{\prime}$ & $\mathrm{Q}^{\prime}$ & $\mathrm{L}^{\prime}$ & $y^{\prime}$ & $\mathrm{J}^{\prime}$ \\
$\mathrm{EA}^{\prime \prime}$ & $\mathrm{D}^{\prime \prime}$ & $\mathrm{Q}^{\prime \prime}$ & $\mathrm{L}^{\prime \prime}$ & $y^{\prime \prime}$ & $\mathrm{J}^{\prime \prime}$ \\
\hline
\end{tabular}

Au lieu de $\left(20^{\prime}\right)$, on a deux conditions :

$$
\left\{\begin{array}{l}
\psi^{\prime} \equiv y+y^{\prime}+h-z^{\prime}-\mathrm{H} \leqslant 0 \\
\psi^{\prime \prime} \equiv y+y^{\prime \prime}+h-z^{\prime \prime}-\mathrm{H} \leqslant 0
\end{array}\right.
$$

avec :

$y=\int_{(\mathrm{L})}^{\mathrm{J}} d x ; \quad y^{\prime}=\int_{\left(\mathrm{L}^{\prime}\right)}^{\mathrm{J}^{\prime}} d x^{\prime} ; \quad y^{\prime \prime}=\int_{\left(\mathrm{L}^{\prime \prime}\right)}^{\mathrm{J}^{\prime \prime}} d x^{\prime \prime}$

Au lieu de (22), on a :

$$
\mathrm{F} \equiv \mathrm{W}+\lambda^{\prime} \psi^{\prime}+\lambda^{\prime \prime} \psi^{\prime \prime}
$$

où $\lambda^{\prime}, \lambda^{\prime \prime}$, sont des paramètres constants, mais inconnus.

On obtient le même $D$, de la conduite de refoulement que par (26). (27) donne :

$$
\lambda^{\prime}+\lambda^{\prime \prime}=\partial W_{2} / \partial H+\left(\partial W_{1} / \partial N\right)(\partial \mathbf{N} / \partial H)
$$

(25) appliguéc aux trois troncons donne, au lieu de $\left(30^{\prime}\right)$, en supprimant les suffixes $k$ :

$$
\left\{\begin{aligned}
\left(\lambda^{\prime}+\lambda^{\prime \prime}\right) \delta \omega^{-1} \mathrm{~K} b^{-1} & =(\mathbf{B} \overline{\mathrm{D}})^{\delta+\omega} \mathrm{Q}^{-m} \\
\lambda^{\prime} \delta \omega^{-1} \mathrm{~K} b^{-1} & =\left(\mathrm{B}^{\prime} \overline{\mathrm{D}^{\prime}}\right)^{\delta+\alpha} \mathrm{Q}^{\prime-m} \\
\lambda^{\prime \prime} \delta \omega^{-1} \mathrm{~K} b^{-1} & =\left(\mathrm{B}^{\prime \prime} \overline{\mathrm{D}^{\prime \prime}}\right)^{\delta+\omega} \mathrm{Q}^{\prime \prime-m}
\end{aligned}\right.
$$

d'où, en éliminant $\lambda^{\prime}, \lambda$,', une condition supplémentaire pour chaque noud :

$(\mathrm{B} \overline{\mathrm{D}})^{\delta+\omega} \mathrm{Q}^{-m}=\left(\mathrm{B}^{\prime} \overline{\mathrm{D}}^{\prime}\right)^{\delta+\omega} \mathrm{Q}^{\prime-m}+\left(\mathrm{B}^{\prime \prime} \overline{\mathrm{D}}^{\prime \prime}\right)^{\delta+\omega} \mathrm{Q}^{\prime \prime-m}$

Pour des grosses conduites, on a approximativement, en vertu de $\left(30^{\prime \prime}\right)$ :

$$
\begin{gathered}
\mathrm{D}_{0}{ }^{\delta+\omega} \mathrm{Q}^{-m}=\mathrm{D}_{60^{\prime+\omega}} \mathrm{Q}^{-m}+\mathrm{D}^{\prime \prime}{ }^{\delta+\omega} \mathrm{Q}^{\prime \prime-m} \\
(\delta+\omega=6,37 ; \quad m=1,852)
\end{gathered}
$$

$\lambda^{\prime}$ et $\lambda^{\prime \prime}$ sont à déterminer par essai; il faut vérifier (52) et (55) ou (56); puis (53') donne $\mathrm{H}$.

Instead of $\left(20^{\prime}\right)$ we have two conditions : (52)

Instead of (22) we get: (53), where $\lambda^{\prime}, \lambda^{\prime \prime}$ are constant unknown parameters.

The same $\mathrm{D}$, of the delivery conduit is found as by (26). Now (27) becomes: $\left(\mathbf{5 3}^{\prime}\right)$

(25) applied to the three sections gives instead of $\left(30^{\prime}\right)$, suppressing the indices $k$ : (54)

Eliminating $\lambda^{\prime}, \lambda^{\prime \prime}$ we get at each junclion the relationship : (55)

For big conduits, very approximately, by $\left(30^{\prime \prime}\right)$, we obtain : (56), where $\lambda^{\prime}, \lambda^{\prime \prime}$ are to be found by trial. (52) has to be verified, then $(55)$ or $(56)$; finally $\left(53^{\prime}\right)$ gives $H$. 
S'il y a plus d'une ramification en $\mathrm{E}$, il faut ajouter les termes correspondants en (55) ou (56).

Lorsque $Q_{0}^{\prime}=Q^{\prime \prime}{ }_{0}$, comme $Q_{0} \geqslant 2 Q_{0}^{\prime}$, on a :

$$
0,277 \mathrm{D}_{0}^{6,37} \geqslant\left(\mathrm{D}_{0}^{\prime 6,37}+\mathrm{D}_{0}^{\prime \prime}{ }^{6,37}\right)
$$

Lorsqu'encore $L^{\prime}=L^{\prime \prime}$ et $z^{\prime}=z^{\prime \prime}$ :

$$
\mathrm{D}_{0}^{\prime} \leqslant 0,733 \mathrm{D}_{0}
$$

Done pour $\mathrm{D}_{0}=400 \mathrm{~mm}, \mathrm{D}_{0}^{\prime} \leqslant 293 \mathrm{~mm}$.

26. - Résean maillé. Soit une conduite principale A EMS avec une maille EM (fig. 10).
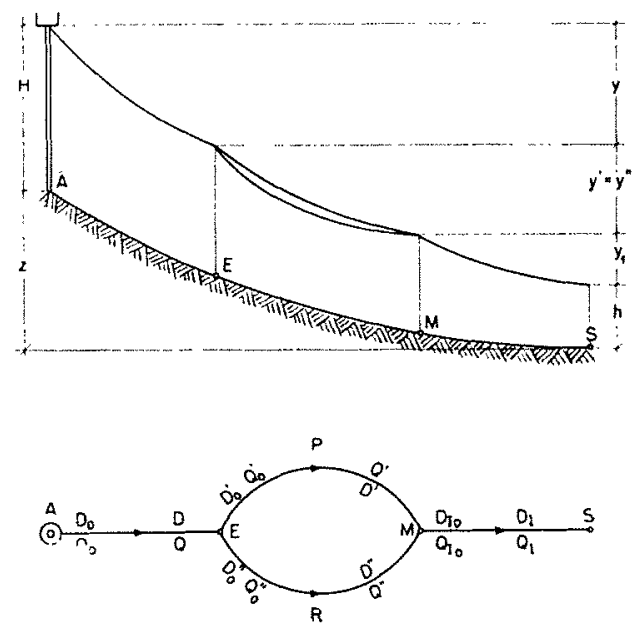

Fig. 10, - Ríseav malulí.

\begin{tabular}{l|c|c|c|c|c}
\hline Tronçon & Diamètre & Débit & Longueur & Perte & Pente \\
\cline { 1 - 3 } A E & $\mathrm{D}$ & $\mathrm{Q}$ & $\mathrm{L}$ & $y$ & $\mathrm{~J}$ \\
$\mathrm{M} \mathrm{S}$ & $\mathrm{D}_{\mathrm{I}}$ & $\mathrm{Q}_{\mathrm{I}}$ & $\mathrm{L}_{\mathrm{I}}$ & $y_{\mathrm{I}}$ & $\mathrm{J}_{\mathrm{I}}$ \\
E P M & $\mathrm{D}^{\prime}$ & $\mathrm{Q}^{\prime}$ & $\mathrm{L}^{\prime}$ & $y^{\prime}$ & $J^{\prime}$ \\
E R M & $\mathrm{D}^{\prime \prime}$ & $\mathrm{Q}^{\prime \prime}$ & $\mathrm{L}^{\prime \prime}$ & $y^{\prime \prime}$ & $\mathrm{J}^{\prime \prime}$ \\
\hline
\end{tabular}

If there are several branches at $\mathrm{E}$, corresponding terms are to be added in (55) or (56).

When $\mathrm{Q}_{0}^{\prime}=\mathrm{Q}_{0}^{\prime \prime}$, as $\mathrm{Q}_{0} \geqslant 2 \mathrm{Q}_{0}^{\prime}$, we have: (57)

when further $L^{\prime}=L^{\prime \prime} ; z^{\prime}=z^{\prime \prime}$, we have : (58)

When $\mathrm{D}_{\theta}=400 \mathrm{~mm}, \mathrm{D}_{i}^{\prime} \leqslant 293 \mathrm{~mm}$.

26. Complex network.-Similar considerations give a new
Au lieu de $\left(20^{\prime}\right)$ on a deux conditions :

$$
\left\{\begin{array}{c}
\psi=y+y^{\prime}+y_{1}+h \ldots-z \cdots H \leqslant 0 \\
y^{\prime}-y^{\prime \prime}=0
\end{array}\right.
$$

avee :

$$
y=\int_{(\mathrm{L})}^{\cdot} \mathrm{J} d x ; \quad y_{\mathrm{I}}=\int_{(\mathrm{L} / \mathrm{l})}^{\mathrm{J}_{\mathrm{l}}} d x_{\mathrm{I}} ;
$$

$$
y^{\prime}=\int_{\left(\mathrm{L}^{\prime}\right)}^{\mathrm{J}} \mathrm{J}^{\prime} d \mathrm{x}^{\prime} ; \quad y^{\prime \prime}=\int_{\left(\mathrm{x} \mathrm{S}^{\prime \prime}\right)}^{\mathrm{J}^{\prime \prime}} d \mathrm{x}^{\prime \prime}
$$

Au lieu de (22), on a :

$$
\mathrm{F} \equiv \mathrm{W}+\lambda \psi+\mu\left(y^{\prime}-y^{\prime \prime}\right)
$$

où $\lambda$, 2 sont des paramètres constants, mais inconnus.

On obtient le même $\mathrm{D}$, de la conduile de refoulement par (26).

(27) donne :

$$
\partial W_{2} / \partial H=\lambda
$$

(25) appliquée aux quatre tronçons donne, en supprimant les suffixes $k$ :

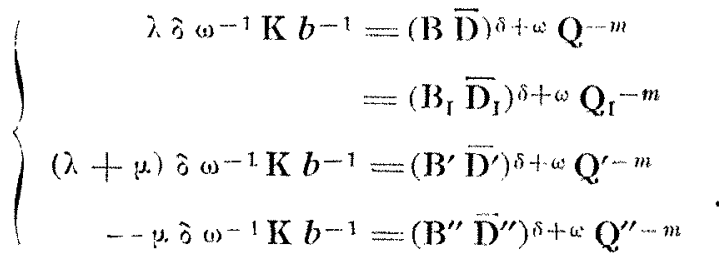

d'où une condition supplémentaire pour chaque. maille du réseau :

$$
\begin{aligned}
& (\mathrm{B} \overline{\mathrm{D}})^{n+\omega} \mathrm{Q}^{-m}=\left(\mathbf{B}_{1} \overline{\mathrm{D}}_{\mathrm{I}}\right)^{i+\omega} \mathbf{Q}_{\mathbf{S}^{-m}} \\
& =\left(\mathbf{B}^{\prime} \ddot{\mathbf{D}^{\prime}}\right) \hat{c}+\omega \mathbf{Q}^{\prime-m}+\left(\mathrm{B}^{\prime \prime} \dddot{\mathrm{D}}^{\prime \prime}\right)^{\delta+\omega} \mathrm{Q}^{\prime \prime-m}
\end{aligned}
$$

Pour de grosses conduites on a approximativement, en verlu de $\left(30^{\prime \prime}\right)$ :

$$
\begin{aligned}
\mathrm{D}_{0}{ }^{\delta+\omega} \mathrm{Q}^{-m} & =\mathrm{D}_{\mathrm{I1}}{ }^{\delta+\omega} \mathrm{Q}_{\mathrm{I} 0^{\delta-m}} \\
& =\mathrm{D}_{0}^{\prime}{ }^{\delta+\omega} \mathrm{Q}_{0}^{\prime-m}+\mathrm{D}^{\prime \prime}{ }^{s+\omega} \mathrm{Q}^{\prime \prime}{ }_{0}^{-m} \\
\delta+\omega & =6,37 \quad m=1,852
\end{aligned}
$$

identique à l'équation d'un réseau ramifié (56). De mème (57), (58) restent valables.

relationship per each circuit : (63)

For large conduits very approximately by $\left(30^{\prime \prime}\right)$; we have: (64)

identical with (56) for a branching network. Also (57), (58) maintain their validity. 
27. - Parfois la maille est une boucle fermée (iig. 11). C'est le cas où $Q_{I}=0$. Dans ce cas, l'emplacement de $M$ est une inconnue supplé-

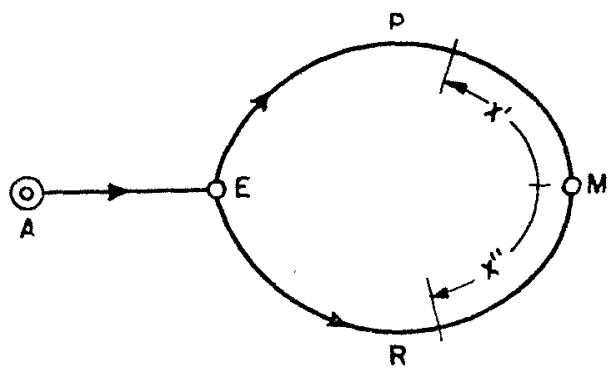

Fiti. 11, - Bovcle.

mentaire. Mesurons $x^{\prime}$ le long de M P E à partir de M et $x^{\prime \prime}$ le long de M R E. Au lieu de $\left(20^{\prime}\right)$, on a quatre conditions:

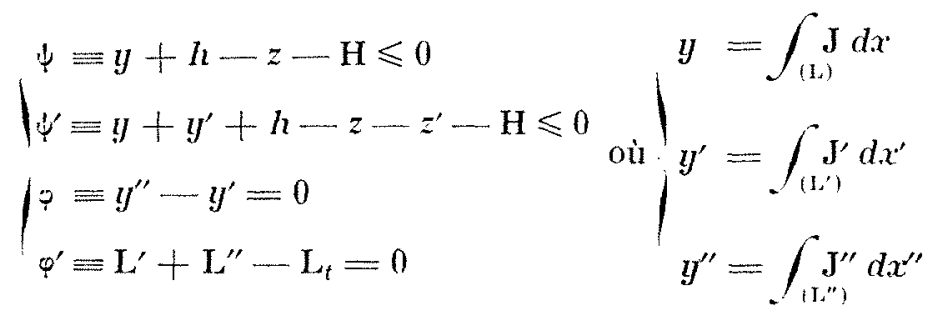

et $\mathrm{L}_{t}$ est la longueur totale de la boucle. Ici $\mathrm{L}^{\prime}, \mathrm{L}^{\prime \prime}$ sont des inconnues supplémentaires; $z^{\prime}$ est fonetion de $\mathrm{L}^{\prime}$ ou $\mathrm{L}^{\prime \prime}$ avec $d z^{\prime} / d \mathrm{~L}^{\prime}=-d z^{\prime \prime} / d \mathrm{~L}^{\prime \prime}$. Multiplions ces équations respectivement par $u, \mu^{\prime}, \lambda, \lambda^{\prime}$, et additionnons-les à $W$. On a, au lieu de (22):

$$
\mathrm{F} \equiv \mathrm{W}+\mu \psi+\mu^{\prime} \psi^{\prime}+\lambda \varphi+\lambda^{\prime} \varphi^{\prime}
$$

D, reste le mème en vertu de (26).

(27) donne :

$$
\mu+u^{\prime}==\partial W_{g} / \partial H+\left(\partial W_{1} / \partial N\right)(\partial N / \partial H)
$$

(25) appliquée aux trois tronçons donne, en supprimant les suffixes $k$ :

$\left(\mu+\mu^{\prime}\right) \delta \omega^{-1} \mathbf{K} b^{-1}=(\mathbf{B} \overline{\mathrm{D}})^{\delta+\omega} \mathbf{Q}^{-m}=\mathbf{D}_{0}^{\delta+\omega} \mathbf{Q}^{-m}$ $\left(u^{\prime}-\lambda\right) \delta \omega^{-1} \mathrm{~K} b^{-1}=\left(\mathbf{B}^{\prime} \overline{\mathbf{D}}^{\prime}\right)^{\delta+\omega} \mathbf{Q}^{\prime-m}={\mathbf{D}_{0}^{\prime}}^{\delta+\omega} \mathbf{Q}_{0}^{\prime-m}$

$$
\lambda \delta \omega{ }^{-1} \mathrm{~K} b^{-1}=\left(\mathbf{B}^{\prime \prime} \overline{\mathbf{D}}^{\prime \prime}\right)^{\delta+\omega} \mathbf{Q}^{\prime \prime-m}=\mathbf{D}^{\prime \prime}{ }_{0}{ }^{\delta+\omega} \mathbf{Q}^{\prime \prime}{ }_{0}{ }^{-m}
$$

$\partial \mathrm{F} / \partial \mathrm{L}^{\prime}=0$ et $\partial \mathrm{F} / \partial \mathrm{L}^{\prime \prime}=0$ donnent :

$\left\{\begin{array}{l}\lambda^{\prime}+\mathrm{P}_{0}^{\prime}+\left(\mu^{\prime}-\lambda\right) \mathrm{J}_{0}^{\prime}-\mu^{\prime} d z^{\prime} / d \mathrm{~L}^{\prime}=0 \\ \lambda^{\prime}+\mathrm{P}_{0}^{\prime \prime}+\lambda \mathrm{J}_{0}^{\prime \prime}-\mu^{\prime} d z^{\prime} / d \mathrm{~L}^{\prime \prime}=0\end{array}\right.$

$\mathrm{D}_{0}^{\prime}, \mathrm{Q}_{0}^{\prime}, \mathrm{J}_{0}^{\prime} ; \mathrm{D}_{0}^{\prime \prime}, \mathrm{Q}_{0}^{\prime \prime}, \mathrm{J}_{0}^{\prime \prime}$ sont les valeurs de $D, Q, J$ près du noud $E$ dans les deux branches. L'élimination de $\lambda, \lambda^{\prime}, \mu, \mu^{\prime}$ des équations (68), $\left(68^{\prime}\right)$, donne :

$$
\mathrm{D}_{0}^{\prime}=\mathrm{D}_{n}^{\prime \prime}
$$

c'est-à-dire pratiquement l'égalité des deux troncons de la boucle. $\mathrm{M}$, qui est le point de débit nul, divise alors la boucle en deux parties égales; (58) reste valable, donc :

$$
\mathrm{D}_{0}^{\prime}=\mathrm{D}_{0}^{\prime \prime} \leqslant 0,753 \mathrm{D}_{0}
$$

\section{VI. - MINIMUM DES DEPENSES ANNUELLES}

28. - Jusqu'ici, nous n'avons parlé que du minimum des capitaux investis. Ce qui nous intéresse davantage, e'est le minimum des dépenses annuelles. Dans ce cas, tous les résultats précédents restent valables, aux coefficients près. En effet, dans le cas traité au chapitre III, on a comme dépenses annuelles :

$$
\min \mathrm{U}=\mathrm{U}_{j}+\mathrm{\Sigma}\left(r+r_{j}+p_{j}\right) \mathrm{W}_{j}+\mathrm{Net}
$$

27. If the circuit is a closed loop (fig. 11) with $Q_{1}=0$, we get by similar considerations: (65)

$$
\mathrm{D}_{0}^{\prime}=\mathrm{D}^{\prime \prime}
$$

or practically the equality of the two portions of the loop. $\mathrm{M}$ is the point of zero discharge which divides the loop into two equal parts; (58) remains valid, so :

$$
\mathrm{D}_{o}^{\prime}=\mathrm{D}^{\prime \prime}{ }_{0} \leqslant 0.733 \mathrm{D}_{0}
$$

\section{VI.-MINIMUM OF ANNUAL EXPENDITURE}

28. Until now, we considered only the minimum of capital investment. The minimum of annual expenditure $U$ is more avec :

$$
\begin{aligned}
& \downarrow \equiv h-z-\mathrm{H}-y_{r}=h-z-\mathrm{H}-\mathrm{J}_{r} \mathrm{~L}_{r} \\
& =h-z-\mathrm{H}-\mathrm{K} \mathrm{Q}^{m}{ }_{r} \mathrm{D}_{r}-i \quad \mathrm{~L}_{r} \leqslant 0 \\
& \text { (6), (17), (18): } \\
& \mathrm{N}=\gamma \mathrm{Q}_{r} \eta^{-1}\left(h_{r}+y_{r}\right) \\
& =\gamma^{r_{s}-1} \mathrm{Q}_{r}\left(h_{s}+\mathrm{KQ}_{r}{ }^{m} \mathrm{D}_{r}{ }^{-\delta} \mathrm{L}_{r}+h_{c}+\mathrm{H}\right)
\end{aligned}
$$

interesting. All previous results remain valid, except the coefficients. Returning to chapter III we get :

$$
\min U=U_{i j}+\mathbf{S}\left(r+r_{j}+p_{j}\right) W_{j}+N e t
$$

with :

$$
\begin{aligned}
\psi & \equiv h-z-\mathrm{H}-y_{r}=h-z-\mathrm{H}-\mathrm{J}_{r} \mathrm{~L}_{r} \\
& =h-z-\mathrm{H}-\mathrm{KQ}_{r}{ }^{\mathrm{m}} \mathrm{D}_{r}{ }^{-\delta} \mathrm{L}_{r} \leqslant 0
\end{aligned}
$$

(6), (17), (18) give $\mathrm{N}=\mathrm{Q}_{r} \cdot \gamma / \eta_{1} \cdot\left(h_{r}+y_{r}\right.$ )

$$
=\mathrm{Q}_{r} \gamma / \eta \cdot\left(h_{s}+\mathrm{KQ}^{\mathrm{m}}{ }_{r} \mathrm{D}_{r}^{-\gamma} \mathrm{L}_{r}+h_{c}+\mathrm{H}\right)
$$


C'est encore un problème de minimum relatif, dont la solution est donnce par le minimum de la fonction auxiliaire $G$ :

$$
\mathbf{G}=\mathbf{U}+\lambda_{1} \psi
$$

$\lambda_{1}$ étant un paramètre inconnu à déterminer. Au lieu de (26), on a :

$$
\begin{aligned}
& \partial \mathrm{G} / \partial \mathrm{D}_{r}=\left(r+r_{3}+p_{3}\right) \partial \mathrm{W}_{3} / \partial \mathrm{D}_{r} \\
& +\left[\left(r+r_{1}+p_{1}\right)\left(\partial \mathrm{W}_{1} / \partial \mathrm{N}\right)+e t\right] \partial \mathrm{N} / \partial \mathrm{D}_{r}=0
\end{aligned}
$$

en vertu de (11), (12), d'où, finalement, au lieu de (29), le diamètre économique $\mathrm{D}_{r}^{\prime}$ de la conduite d'adduction :

$$
\begin{gathered}
\mathrm{D}_{r}^{\prime}=\beta^{\prime}{ }_{w} \mathrm{Q}_{r}{ }^{\prime \prime} \quad u=(m+1) /(\omega+\delta)=0,447 ; \\
1 /(\omega+\delta)=0,157 \\
\beta^{\prime}{ }_{r r}=\beta_{w} \frac{\left[\left(r+r_{1}+p_{1}+e t b^{-1}\right)\right.}{\left.\left(r+r_{3}+p_{3}\right)\right]^{1 /(\omega+\delta)}}
\end{gathered}
$$

$\mathrm{D}_{r}^{\prime}$ est encore indépendant de la longueur $\mathrm{I}_{r}$ de la conduite d'adduction. Comme aussi :

$$
\begin{aligned}
& \beta^{\prime}{ }_{w}>\beta_{10} \\
& \mathrm{D}_{r}^{\prime}>\mathrm{D}_{r} ; \quad v_{r}^{\prime}<v_{r} ; \quad \mathbf{J}_{r}{ }_{r}<\mathbf{J}_{r}
\end{aligned}
$$

c'est-à-dire que le minimum des dépenses annuelles exige une conduite d'adduction plus grosse que ne l'exige le minimum des capitaux.

29. - En appliquant (25), on obtient pour la conduite principale les mêmes équations (30)(41), mais au lieu de $\lambda$ il faut écrire partout $\lambda /\left(r+r_{4}+p_{4}\right)$, et au lieu de $b_{1}$ ćcrire :

$$
b_{1}\left(r+r_{1}+p_{1}\right) /\left(r+r_{4}+p_{4}\right),
$$

où, en général, $r_{4}=r_{3}$ et $p_{4}=p_{3}$.

30. - En appliquant (27) selon la méthode du chapitre IV, on obtient les formules (43) dans lesquelles il faut remplacer $\lambda / b$ par :

$$
\frac{b_{22}\left(r+r_{2}+p_{2}\right)+b_{1} \gamma^{-1} \mathrm{Q}_{r}\left(r+r_{1}+p_{1}\right)}{\vec{b}\left(r+r_{4}+p_{4}\right)}
$$

This is again a problem of relative minimum, the solution of which is given by the minimum of the auxiliary function $G$.

$$
\mathrm{G}=\mathrm{U}+\lambda_{1} \mathrm{y}
$$

$\lambda_{1}$ is an unknown parameter to be determined. (26) is replaced by : $\partial \mathrm{G} / \partial \mathrm{D}_{r}=\left(\mathrm{r}+\mathrm{r}_{3}+p_{3}\right) \partial \mathrm{W}_{3} / \partial \mathrm{D}_{r}+$

$\left[\left(r+r_{1}+p_{1}\right) \partial W_{1} / \partial \mathrm{N}+c t\right] \partial \mathrm{N} / \partial \mathrm{D}_{r}=0$ by $(11),(12)$.

So finally instead of (29) we have the economical diameter $\mathrm{D}^{\prime}{ }_{r}$ of the delivery conduit : (70)

$D_{r}^{\prime}$ is again independent of the length $L_{r}$ of the conduit. Also : (71)

$\mathrm{D}_{r}^{\prime}>\mathrm{D}_{r} ; v_{r}^{\prime}<v_{r} ; \mathrm{J}_{r}^{\prime}<\mathrm{J}_{r}$ and the minimum of annual expenditure requires a larger delivery conduit than the minimum of capital investment.
Comme $r_{2}+p_{2}<r_{4}+p_{4}$, et $r_{1}+p_{1}>r_{4}+p_{4}$, l'expression (72) est sensiblement égale à $b_{2} / b$, et d'après (43) :

$\mathrm{D}_{0}^{\prime} \approx \mathrm{D}_{0} ; \quad v_{0}^{\prime} \approx v_{0} ; \quad \mathrm{J}_{0}^{\prime} \approx \mathrm{J}_{0} ; \quad \mathrm{H}^{\prime} \approx \mathrm{H}$

où l'apostrophe se rapporte au minimum des dépenses annuelles; d'où la règle suivante :

L'économie de prix de revient (ou des dépenses annuelles) nécessite une conduite d'adduction plus grosse et, sensiblement, même conduite principale et même hauteur du réservoir que dans le cas du minimum des capitaux.

31. - Dans le cas du paragraphe 23, il suffii d'écrire, au lieu de (48) pour $\varepsilon=1$ :

$$
\begin{aligned}
\Delta W & =\left(r+r_{2}+p_{2}\right)\left(a_{2}+b_{2} \mathrm{H}\right) \\
& +\left(r+r_{3}+p_{3}\right) \mathrm{P}_{0} \xi \\
& +b_{1} \gamma / \eta \cdot \mathrm{Q}_{1} \mathrm{H}\left(r+r_{1}+p_{1}\right)+\mathrm{N}(\mathrm{H}) \rho t \\
& <\left(r+r_{2}+p_{2}\right)\left(a_{2}+b_{2} \mathrm{H}_{0}\right) \\
& +b_{1} r / \eta \cdot \mathrm{Q}_{2} \cdot \mathrm{H}_{0}\left(r+r_{1}+p_{1}\right)+\mathrm{N}\left(\mathrm{H}_{01}\right)
\end{aligned}
$$

ct la pente critique économique devient, au lieu de (50):

$$
\begin{aligned}
\mathrm{J}_{r r}^{\prime}= & \mathrm{J}_{0}+\frac{\mathrm{P}_{0}\left(r+r_{2}+p_{3}\right)}{\left[e l+b_{1}\left(r+r_{1}+p_{1}\right)\right] \gamma r^{-1} \mathrm{Q}_{r}+b_{2}\left(r+r_{2}+\right.} \\
& <\mathrm{J}_{\mathrm{cr}}
\end{aligned}
$$

La pente critique est heaucoup plus fable que seion $(50)$.

32. - - Il en est de même lorsqu'on tient comple des conduiles sceondaires du réseau ramifié ou maillé. On obtient les mêmes formules que dans le cas du minimum des capitaux.

29. Applying (25), we get for the main the same equations (30) - (41), but $\lambda$ is replaced by $\lambda /\left(r+r_{4}+p_{4}\right) ; b_{1}$ by $b_{1}\left(r+r_{1}+p_{1}\right) /\left(r+r_{4}+p_{4}\right)$. Usually $r_{4}=r_{3}$ and $p_{4}=p_{3}$.

30. Applying (27) and the method of chapter IV, we get (43) where $\lambda / b$ is replaced by : (72)

As $r_{2}+p_{2}<r_{4}+p_{4}$ and $r_{1}+p_{1}>r_{4}+p_{4}$, this expression is approximately $b_{2} / b$ and by (43), we obtain : (73) where the apostrophe refers to the minimum of annual expenditure. Hence the following rule :

The economy of the price of water (or of the annual expenditure) requires a larger delivery conduit than, and sensibly the same main and height of water tower, as the condition of minimurn of capital investment.

31, 32. Please refer to the French text. 
33. -... Pour terminer, il faut remarquer qu'il ne faut pas chercher une grande précision puisque :

a) Au voisinage d'un minimum, Ia fonction varie fort peu, même lorsque les variables s'écartent du minimum;

b) La plupart des formules sont empiriques et les coefficients $m, \hat{o}, \omega, \varepsilon, a_{1}, b_{1}, a_{2}, b_{2}$, $a_{3}, b_{3}, a, b$, etc., ne sont connus qu'à peu près; c) Les diamètres ne peuvent varier que par bonds et ne varient en général que comme une faible puissance des débits et des prix.

34. - Cette méthode peut encore s'appliquer aux réseaux électriques de distribution. La méthode développée ci-dessus est enseignéc par l'auteur à l'Israel Institute of Technology, Haifa, depuis 1945. Il s'est avéré qu'elle donne des ré sultats rapides ef exacts en peu de temps.

\section{A P PE N D C E}

\section{APPENDICE A}

\section{Valeurs de la durée de vie $T_{j}$} et du taux d'amortissement annuel $r_{j}$ au taux $r=0,05(5 \%)$

EN ISRAEL, EN 1950 [2] :

1 - Filtre du puits; pompe à plongeur; éléments de machines; instruments........

2 - Moteur Diesel rapide; compteur d'eau..............

3 - Pompe verticale immergée, pompe horizontale (aspiration $5 \mathrm{~m}) \ldots \ldots \ldots \ldots \ldots \ldots$

4 -.- Moteur Diesel lent; pompe horizontale (aspiration $<5 \mathrm{~m}$ ); conduites secondaires non protégées; appareils de traitement de l'eau...........

5 -. Pompe à piston...........

6 - Puits foré; conduite principale non protégie; moteur électrique..$\ldots \ldots \ldots \ldots$.

$\underset{\mathrm{T}_{j}}{\operatorname{ANs}} r_{i}$

20,1047

$10 \quad 0,0795$

120,0628

$15 \quad 0,0463$

$18 \quad 0,0355$

$20 \quad 0,0302$

33. Finally, we may notice that no great precision is required, as :

a) Near a minimum the function varies slowly, even when the variables deviate considerably from the minimum.

b) Most formulae are empirical and the values of the coefficients $m, \delta, \omega, \varepsilon, a_{1}, b_{1}, a_{2}, b_{2}, a_{3}, b_{3}, a, b$, etc. are known only approximately.

c) The diameters can vary only by jumps and only as a small

7 - Puits ordinaire; station de pompage (bâtiment): conduite principale protégée...... $25 \quad 0,0210$

8 - Réservoir en héton....... $40 \quad 0,0083$

\section{APPENDICE B}

Valeurs du taux annuel $p_{j}$ entretien, réparations, assurance [2]

1 - Puits ordinaire....... 0,002

2 - Réservoir ......... $0,001-0,004$

3 - Conduite métallique.... $0,002 \ldots 0,020$

4 -Puits foré et filtre..... 0,001

5 - Moteur électrique...... $0,010 \ldots 0,015$

6 _. Bâtiment .......... $0,010 \ldots 0,020$

7 - Pompe à piston....... $0,020-0,030$

8 - Pompe horizontale. . . . . 0,020 - 0,040

9 - Pompe de forage profond $0,040-0,060$

10 - Moteur Diesel (sans graissage) $\ldots \ldots \ldots \ldots \ldots \ldots 0,030-0,050$

11 - Compteur d'eau ( $>40 \mathrm{~mm}$ ) $0,040-0,060$

12 - Compteur d'eau $(<40 \mathrm{~mm}) \quad 0,100$

13 - Assurance des bâtiments. 0,002-0,003

14 -..- Assurance des machines. . 0,003-0,005

power of the discharge and prices.

34. This method may be applied also to electrical distribution networks.

The method developed here is being laught by the author at the Israel Institute of Technology, Haifa, since 1945. It proved to give rapid and exact results in a short time.

BIBLIOGRAPHY.-Please refer to the French text. 


\section{APPENDICE C}

Justification de l'équation $\mathbf{P}=\boldsymbol{a}+\boldsymbol{b} \mathbf{D}$

L'épaisseur $s$ d'une conduite est :

$$
s=s_{0}+p \mathrm{D} / 2 \text { 。 }
$$

où $s_{0}=1$ à $2 \mathrm{~mm}$ (corrosion); $\sigma=$ effort de tension permis; $p=$ pression hydraulique $; \mathrm{D}=$ diamètre interne de la conduite; $\gamma^{\prime}=$ son poids unitaire. Le poids de la conduite par mètre est donc:

$$
\gamma^{\prime} \pi \mathrm{D} s=\pi \gamma^{\prime} s_{0} \mathrm{D}+(\pi / 2) p\left(\gamma^{\prime} / \sigma\right) \mathrm{D}^{2}
$$

équation d'une parabole. Au prix de la conduite, il faut ajouter les frais suivants : accessoires, enduits, peinture, excavation, mise en place et remblayage.

\section{APPENDICE D}

\section{Calcul de la formule $P=a+b D^{\omega}$}

Les prix $\mathrm{P}_{k}$ sont connus pour divers diametres $\mathrm{D}_{k}$ disponibles. On mène une courbe par tous ces points. Par extrapolation, vers $D=0$, on obtient sur l'axe des $P$ le segment $a$. Puis on écrit :

$$
\log \left(\mathrm{P}_{k}-a\right)=\log b+\omega \log \mathbf{D}_{k}
$$

Par la méthode des moindres carrés, on obtient :

$$
\min \mathbf{F} \equiv \sum_{k=1}^{n}\left[\log b+\omega \log \mathrm{D}_{k}-\log \left(\mathrm{P}_{k}-a\right)\right]=
$$

done :

$$
\partial \mathrm{F} / \partial \log b=0 ; \quad \partial \mathrm{F} / \partial \omega=0
$$

d'où :

$\log b=\left[\Sigma \log \left(\mathrm{P}_{k}-a\right) \cdot \mathrm{x}\left(\log \mathrm{D}_{k}\right)^{2}\right.$

$$
\left.-\Sigma \log \mathrm{D}_{k i} \cdot \log \left(\mathrm{P}_{k}-\alpha\right) \cdot \Sigma \log \mathrm{D}_{k}\right] / \Delta
$$

$\omega=\left[n \Sigma \log \mathrm{D}_{k} \cdot \log \left(\mathrm{P}_{k}-\alpha\right)\right.$

$$
\left.-\mathbf{v} \log \mathrm{D}_{k} . \mathbf{\Sigma} \log \left(\mathrm{P}_{k}-\boldsymbol{a}\right)\right\rceil / \Delta
$$

où :

$$
\begin{aligned}
& \Delta=n \Sigma\left(\log \mathrm{D}_{k}\right)^{2}-\left(\Sigma \log \mathrm{D}_{k}\right)^{2} \\
& n=\text { nombre de diamètres } \mathrm{D}_{k} \text { envisagés. }
\end{aligned}
$$

\section{APPENDICE E}

\section{Formule de Hazen-Williams pour le calcul des conduites} recalculée en unités métriques par l'auteur

\section{Formules}

Vitesse moyenne :

$$
v(\mathrm{~m} / \mathrm{s})=354 \mathrm{Q} \mathrm{D}^{-2}
$$

$$
\begin{aligned}
& =1,096 \cdot 10^{-4} \mathrm{C} \cdot \mathrm{J}^{0,54} \mathrm{D}^{0.63} \\
& =3,97 \cdot 10^{-3} \mathrm{C}^{0.761} \mathrm{Q}^{0,239} \mathrm{~J}^{0.411}
\end{aligned}
$$

Penle d'énergie :

$$
\begin{aligned}
& \mathrm{J}(\% \omega)=1,131.10^{12}(\mathrm{Q} / \mathrm{C})^{1,852} \mathrm{D}^{-4,4 \pi} \\
= & 2,16.10^{7}(\mathrm{v} / \mathrm{C})^{1.852} \mathrm{D}^{-1,16 \pi} \\
= & 7,02.10^{5} \mathrm{C}^{-1,852} v^{2.436} \mathrm{Q}^{-0,584}
\end{aligned}
$$

Débit :

$$
\begin{aligned}
& \mathrm{Q}\left(\mathrm{m}^{3} / \mathrm{h}\right)=2,83 \cdot 10^{-3} v \mathrm{D}^{2} \\
= & 3,1 \cdot 10^{-\tau} \mathrm{CJ} \mathrm{J}^{0,51} \mathrm{D}^{2,63} \\
= & 1,057 \cdot 10^{10}(v / \mathrm{C})^{4,155} \mathrm{~J}^{-1,714}
\end{aligned}
$$

Diamètre intérieur :

$$
\begin{aligned}
& \text { I) }(\mathrm{mm})=18,8 \mathrm{Q}^{0,5} v^{-0,3} \\
= & 298(\mathrm{Q} / \mathrm{C})^{0.38} \mathrm{~J}^{-0,208} \\
= & 1,93.10^{6}(\mathrm{v} / \mathrm{C})^{1,587} \mathrm{~J}^{-0,8 \pi 7}
\end{aligned}
$$

\section{Vherers de complatent $C$}

Materiau

ritat du tuyan

C

ordinaire

Fonte:

neuf centrifugé

neuf, ordinaire

20 ans, caux dures

125-135

120-130

usé (voir table ci-après).

Vulcurs de $\mathrm{C}$

\begin{tabular}{|c|ccccccc|}
\hline $\mathrm{D}$ & \multicolumn{7}{|c|}{ Age en années } \\
\cline { 2 - 8 } $\mathrm{min}$ & 0 & 5 & 10 & 20 & 30 & 40 & 50 \\
\hline 100 & 130 & 118 & 107 & 89 & 75 & 04 & 55 \\
200 & 130 & 119 & 109 & 93 & 83 & 73 & 65 \\
300 & 130 & 120 & 111 & 96 & 86 & 77 & 70 \\
400 & 130 & 120 & 112 & 98 & 87 & 80 & 72 \\
600 & 130 & 120 & 113 & 100 & 89 & 81 & 74 \\
750 & 130 & 120 & 113 & 100 & 90 & 83 & 76 \\
900 & 130 & 120 & 113 & 100 & 90 & 83 & 76 \\
1.000 & 130 & 120 & 113 & 100 & 90 & 83 & 77 \\
1.500 & 130 & 120 & 113 & 100 & 90 & 83 & 77 \\
\hline
\end{tabular}

Acren

Acurn, rivé 


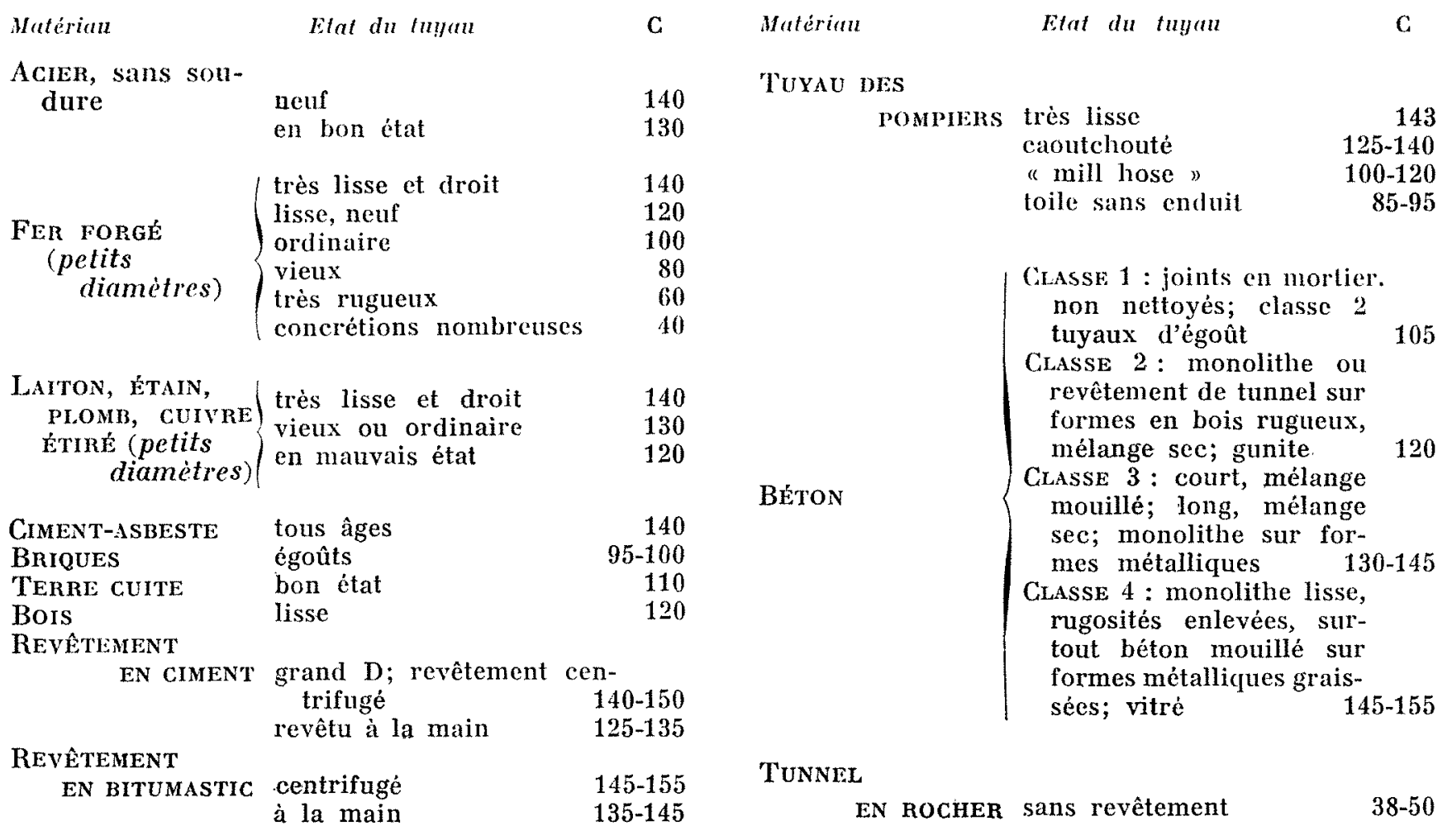

La table qui suit donne pour $\mathrm{C}=100$, qui est une valeur moyenne, les valeurs correspondantes de $\mathrm{D}, \mathrm{Q}, v, J ; 25<\mathrm{D}<1.250 \mathrm{~mm}$; $0,2<\mathrm{Q}<11.000 \mathrm{~m}^{3} / \mathrm{h} ; 0,10<v<7 \mathrm{~m} / \mathrm{s}$; $0,02<\mathrm{J}<4.000 \%$. L'interpolation est aisée, car les différences sont faibles. Lorsque $C$ est différent de 100 , on procède comme suit :

(1) Donnḱes $\mathrm{D}, \mathrm{Q}:$ On tire $v$ de la table (indépendant de $\mathrm{C})$; on calcule $\mathrm{Q}_{0}=\mathrm{Q}(100 / \mathrm{C})$; on tire de la table $\mathrm{J}\left(\mathrm{D}, \mathrm{Q}_{0}\right)$.

(2) Données $\mathrm{D}, \mathrm{J},:$ On tire de la table $Q_{0}$ et $v_{0}$; on les multiplie par $\mathrm{C} / 100$.
(3) Données $Q, J$ : On calcule $Q_{0}=Q(100 / C)$; on tire de la table $\mathrm{D}\left(\mathrm{Q}_{0}, J\right)$ et $v(\mathrm{Q}, \mathrm{D})$.

(4) Données $\mathrm{D}, v$ : On tire de la table $\mathrm{Q}$ (indépendant de $\mathrm{C}$ ); puis on procède comme pour (1).

(5) Données $\mathrm{Q}, v$ : On tire de la table $\mathrm{D}$; puis on procède comme pour (1).

(6) Données $v$, J : On calcule $v_{0}=v(100 / \mathrm{C})$; on tire de la table $\mathrm{D}\left(v_{0}, \mathrm{~J}\right)$ et $\mathrm{Q}(\mathrm{D}, v)$. 


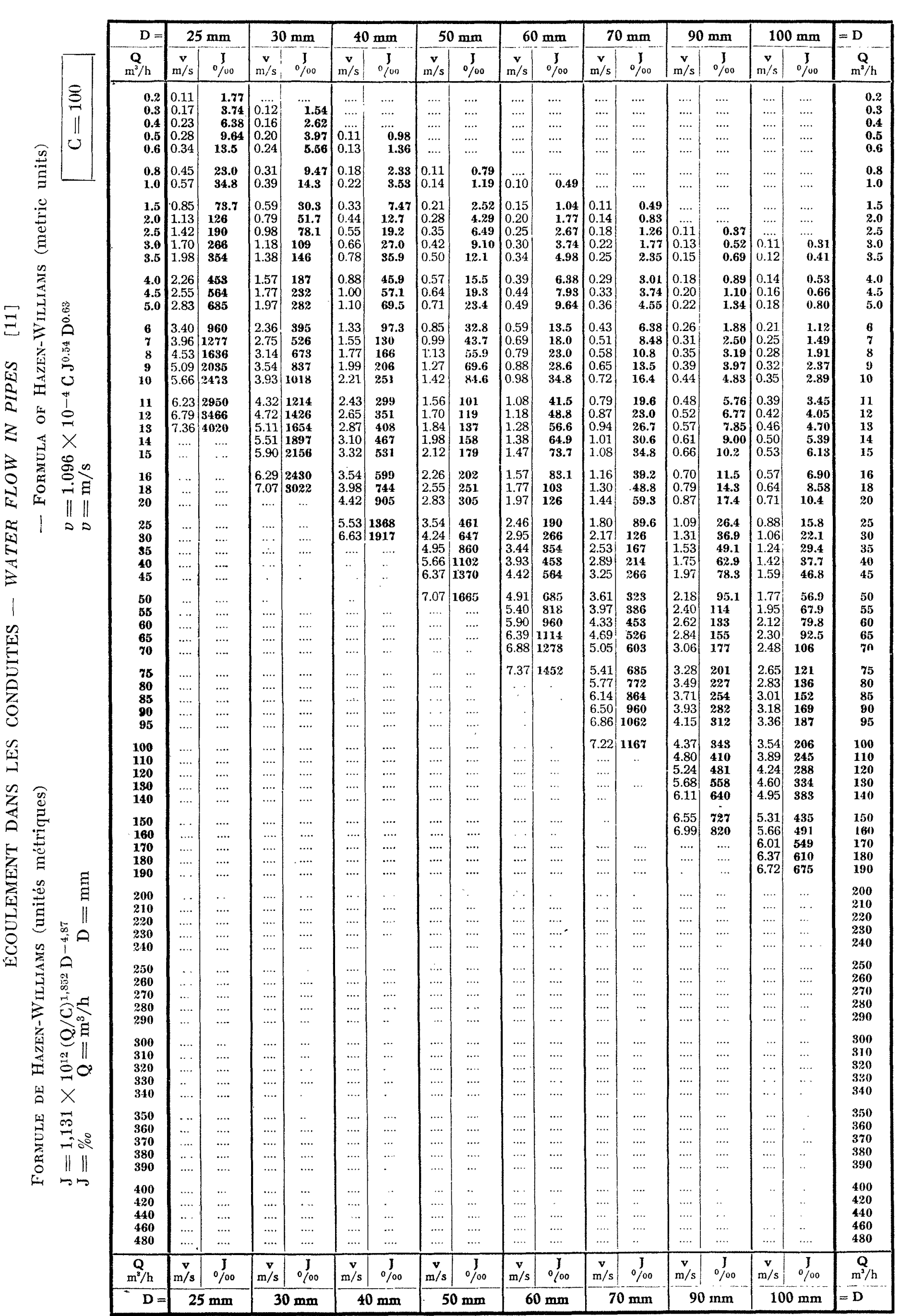




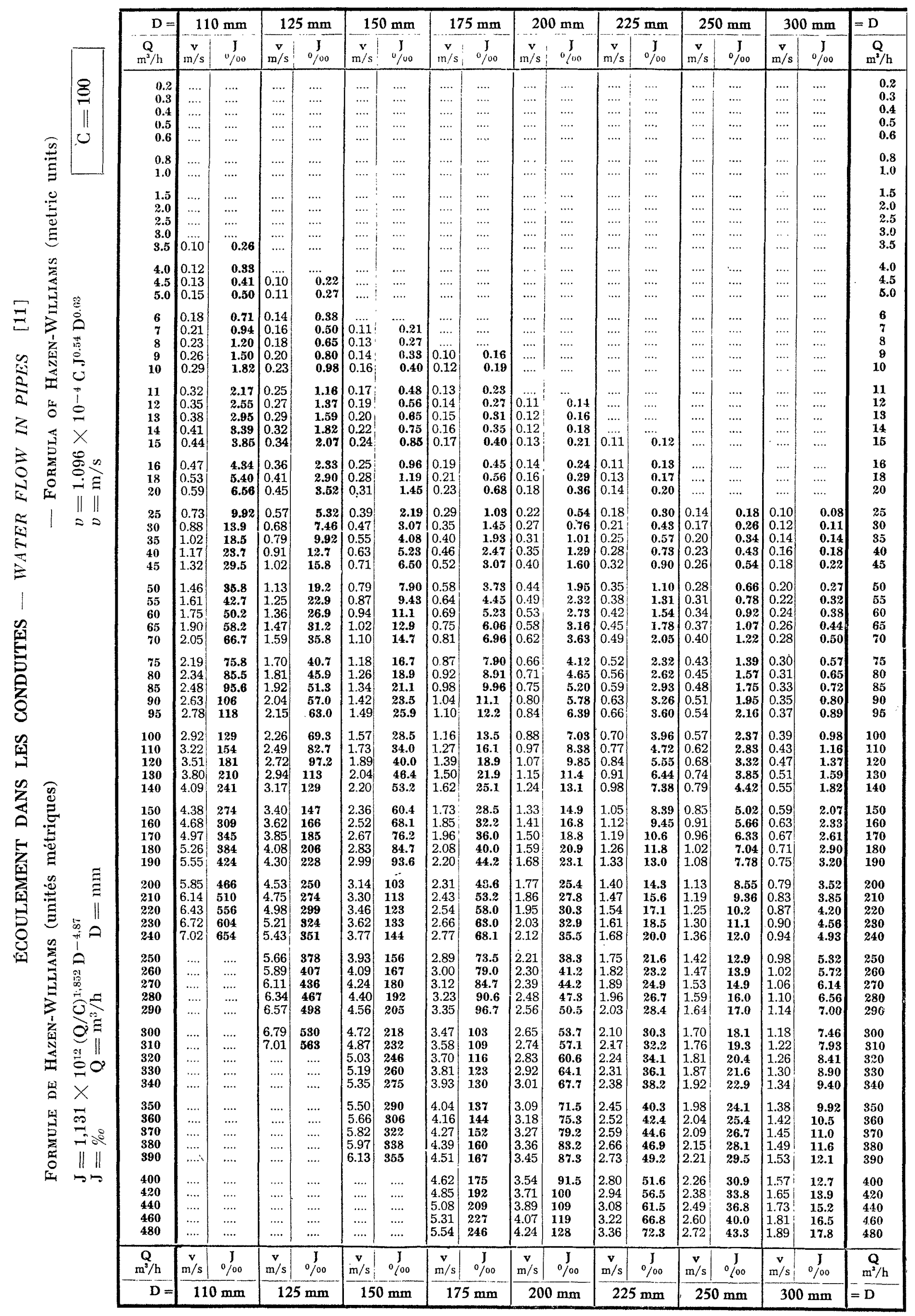









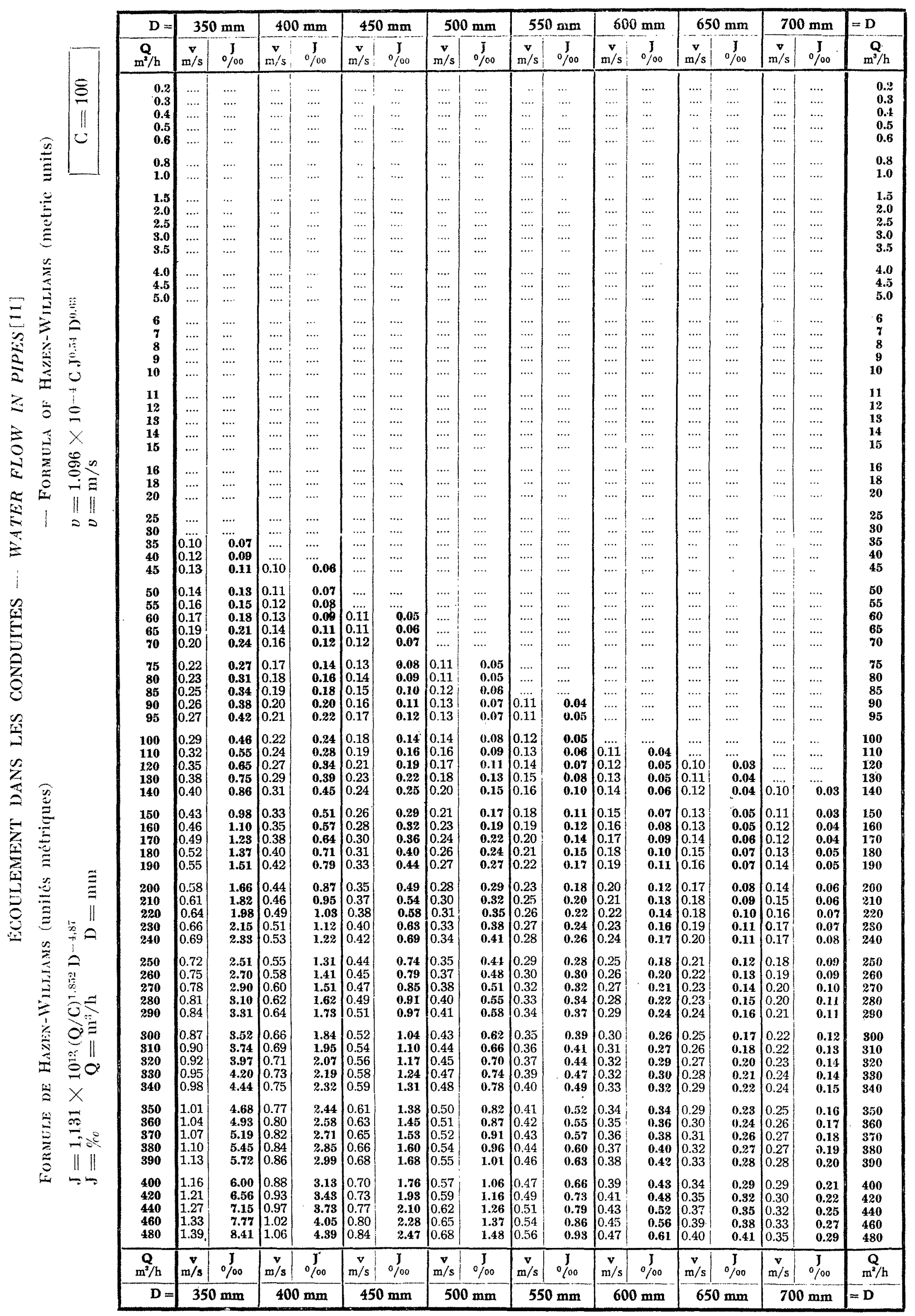




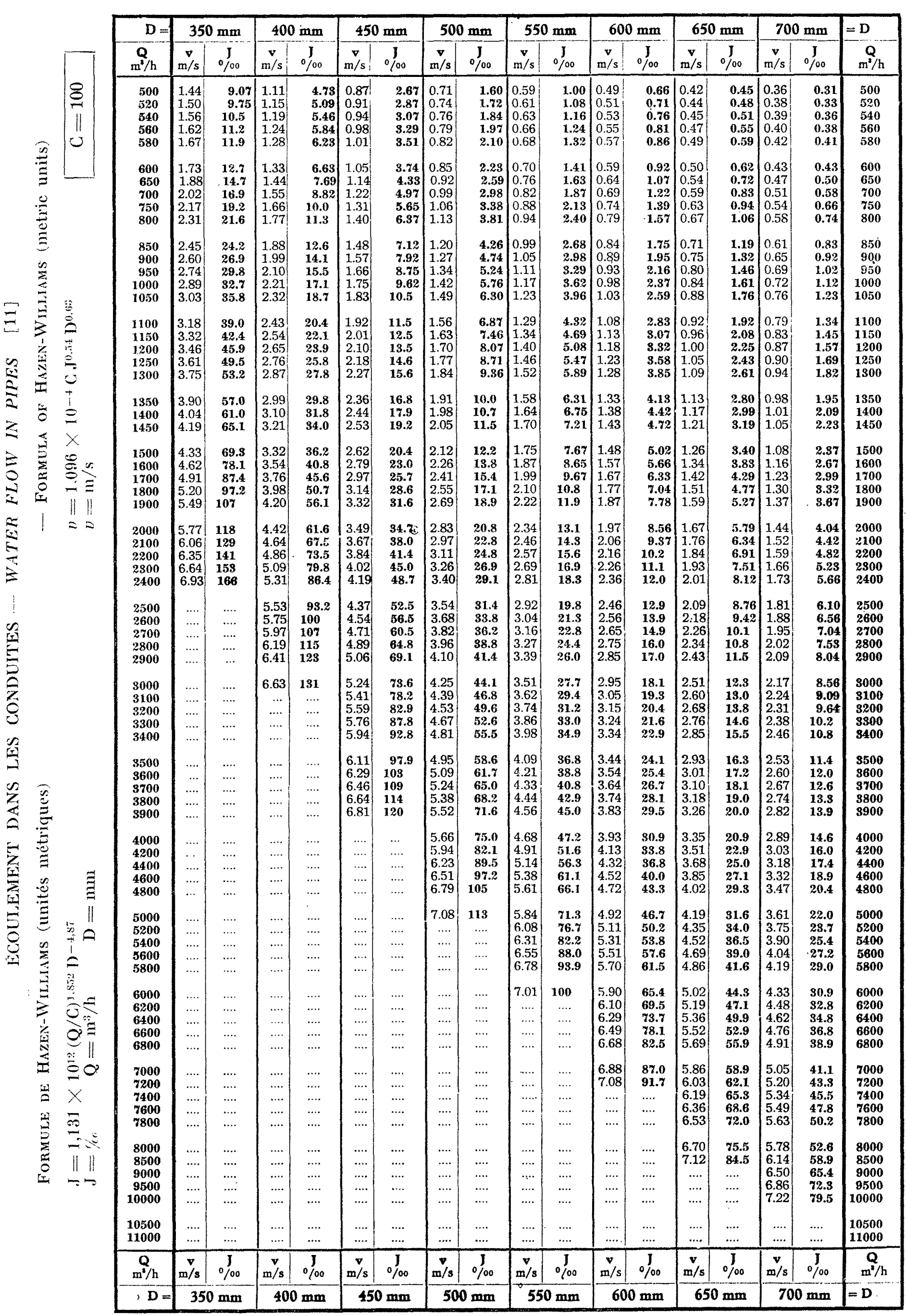




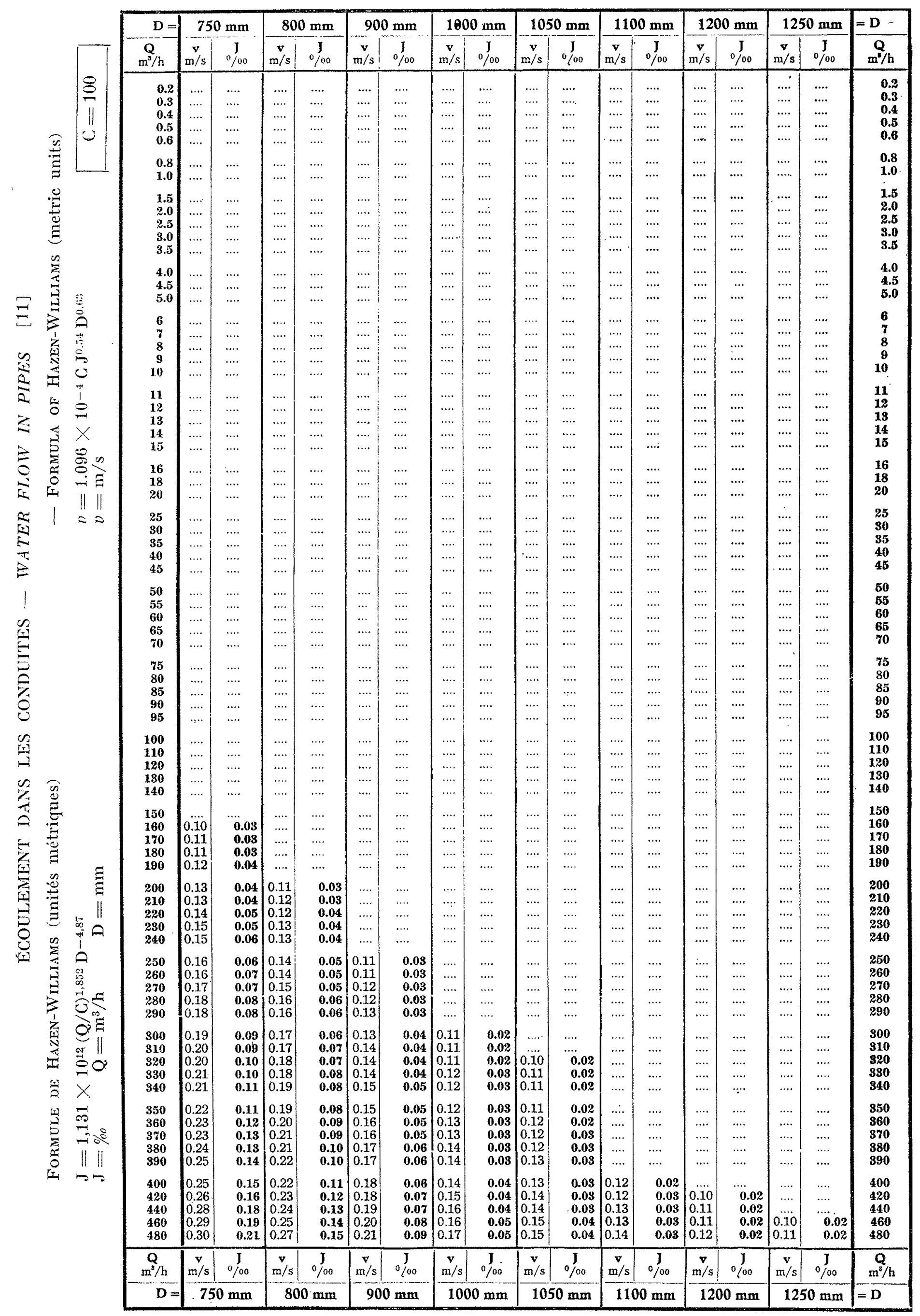




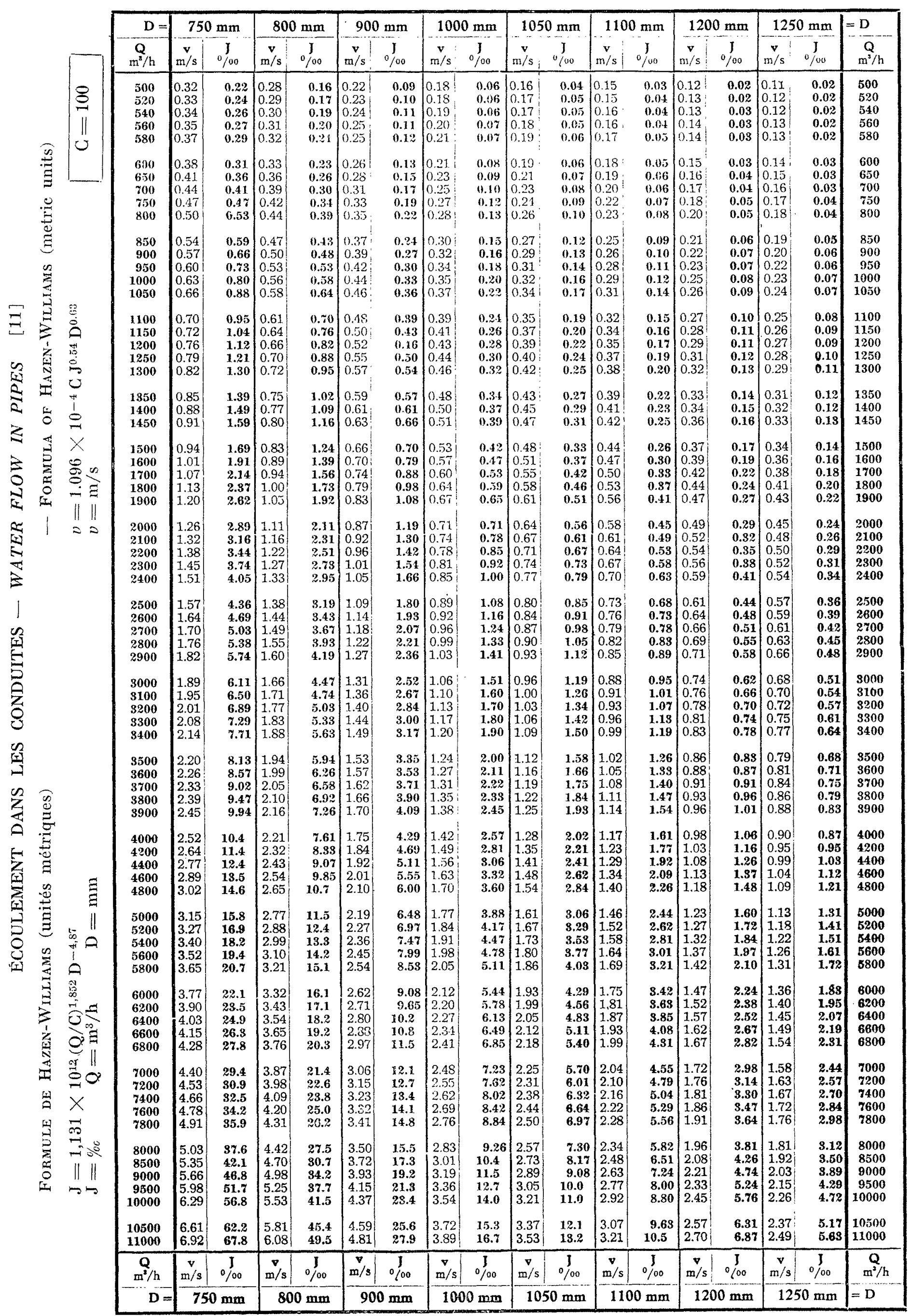




\section{APPENDICE F}

\section{Valeurs de $f$}

Des considérations dimensionnelles montrent que $f$ dépend du régime d'ćcoulement :

1. - En écoulement lisse, il est fonction du nombre de Reynolds $R=b \mathrm{D} / \nu$ (où $\nu=$ riseosité cinématique). Buasies, Karman et Prandtl en ont donné des formules semi-empiriques [6].

2. - En écoulement turbulent rugueux, il est fonction de la rugositi relative $k / D$ ) oit $k=$ hauteur maxima des rugosités, [6]. Nikcradse en a donné une formule semi-empirique.

3. -... En écoulement turbulent intermediaire, il est fonction de $\mathcal{R}$ et $k / D$ à la fois et dépend en outre de la distribution des rugosités. ColsBRoOk el White ont donné une formule empirique valable pour les conduites commerciales [6].

Ces formules serviront a l'atenir comme formules de base. Ici, cependant, nous avons préféré la formule empirique de Hazen Wildiams. Il est curieux cependant de conslater qu'elle correspond pour $\mathrm{C}=130$ (acier lisse) à $f=0,16 \Omega^{-1 / 6}$ a $15^{\circ} \mathrm{C}$ et représente à quelques \% près les mesures jusqu'à $R=20 \times 10^{6}[10]$.

\section{APPENDICE G}

\section{La preuve de l'existence d'un minimum de $\mathrm{W}$}

La fonction $\psi$ doit ètre [5] une fonction continue des $(n-1)$ inconnues $x_{i}$ et ses dérivées partielles par rapport aux $(n-2)$ inconnues $x_{k}$ au moins doivent être continues. Puisque $Q(x)$ n'est, en général, pas une fonction continue de $x$, $J$ et $\psi$ ne le sont pas non plus. Il a fallu recou- rir à l'expédient mentionné ci-dessus et remplacer la courbe à gradins $Q(x)$ par une courbe continue infiniment proche.

Il faul concore que l'expression :

$$
\begin{aligned}
\Delta=\mathrm{F}\left(x_{1}+x_{1}, \ldots x_{n+2}+\right. & \left.x_{n+2}\right) \\
& \quad-F\left(x_{1}, \ldots x_{n ! 2}\right)>0
\end{aligned}
$$

pour des valeurs $\alpha_{k}$ arbitraires, positives ou ncigatives, mais voisines de zéro. Te développement en série de TAYlor donne :

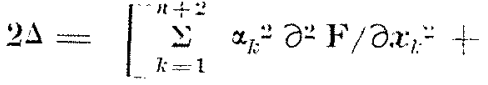

$$
\begin{aligned}
& \left.\sum_{n+2}^{k} \sum_{j=1}^{n+2} 2 \alpha_{k} x_{j} \partial^{2} F / \partial x_{k} \partial x_{j}\right\rfloor+ \text { termes plus petils } \\
& >0
\end{aligned}
$$

Pour $\alpha$ très petits, l'expression entre crochets détermine le signe. Or, d’après (25) :

$\partial^{2} \mathbf{F} / \partial x_{k}{ }^{2}=m \mathbf{Q}_{k}^{m-1}\left(\partial \mathbf{Q}_{k} / \partial x_{k}\right) \cdot \lambda \mathbf{K}\left(\mathbf{D}_{k-1}{ }^{-6} \ldots \mathbf{D}_{k}^{-i n}\right)$

$$
>0(n=1, \ldots n-1)
$$

puisque $\partial \mathrm{Q}_{k} / \partial \boldsymbol{x}_{k}<0 ; \mathrm{D}_{k-1}>\mathrm{D}_{k i} ; \lambda>0$ et sensiblement $K=$ const. De mème, d'après (26), (28),

$$
\begin{gathered}
\partial \% \mathrm{~F} / \partial x_{k}^{\prime}(k=n, n+1, n+2) ;>0 ; \text { aussi : } \\
\partial \div \mathrm{F} / \partial x_{k} \partial x_{j}=0(k \neq \hbar j ; k, j=1, \ldots n+2)
\end{gathered}
$$

Donc $\Delta>0$ et nous avons démontré l'existence d'un minimum vai des capitaux investis, W, donc d'une solution économique.

On démontre de la même façon l'existence d'un ninimum vrai des dépenses annuelles $U$.

\section{Bibliographie}

[1] J. SPRUY : Consommation d'eau domestique et industrielle fournie par un service public de distribution (1\% Congrès 1949, Amsterdam, Assoc. Internat. de Distribution d'eau).

[2] Isracl Standards Institute : Tentative standard for the computation of the price of water (Tel-Aviv $1950)$.

[3] J.S. Corrox: Interim report on master plan for development of irrigation and hydroelectrie power in State of Israel, vol. II (Jérusalem, 1951).

44 C. V. Davss: Handbook of applied hydraulics, p. 732 (NeGraw Hill, New-York, 1942).

[5] E. Gounsat : Cours d'analyse mathématique, tome I, p. 107-122 (Gauthier-Villars, Paris, 1923).
[6] S. Inmay : On steady flow formulae in pipes and channe's (Association Internationale de Recherches pour Traraux Hydrauliques, 3" réunion, Grenoble, 1949).

(a) M. V. Kimsanory: Calcul économique des réseaux de distribulion d'eau (en russe) (Moscou, 1949).

8) P.A.M. PARkER : The control of water, p. 445 (Routledge \& Sons, London, 1932).

[9] A. Scanocklitsch : Der Wasserbau (1938).

[10] R. B. Genereaux : Fluid friction in conduits (Fluid and particle mechanics by C. F. Laprie, Univ. of Delawale, Newark, Delaware, 1951).

[11] S. InmaY : Tables for pipe flow (metric units), formula of Ilazex-Williams (Institute of Technology, Haifa, Israel. 1951). 